\title{
Numerical correlation for natural convective flows in isothermal heated, inclined and convergent channels, for high Rayleigh numbers
}

\author{
A.S. Kaiser, B. Zamora, A. Viedma \\ Dpto. Ingeniería Térmica y de Fluidos, Universidad Politécnica de Cartagena, \\ Doctor Fleming s/n, 30202 Cartagena, Spain
}

\begin{abstract}
A numerical study of natural convective flows, mainly for high Rayleigh numbers, in a sloped converging channel, for different inclination and convergence angles has been carried out, taking into account the lacks of the literature on some aspects of this configuration. Twodimensional, laminar, transitional and turbulent simulations were obtained by solving the fully-elliptic governing equations using two different general purpose codes: Fluent and Phoenics. The low-Reynolds $k-\omega$ turbulence model has been employed. Special emphasis is made on the systematic comparisons of computational results with experimental and numerical data taken from literature for turbulent regime, so as on the transitional conditions, studying the influence of Rayleigh number and channel aspect ratio. A generalized turbulent correlation for the average Nusselt number has been obtained from numerical results in a channel with isothermal heated plates, for symmetric heating conditions. This correlation is valid for wide and not yet covered ranges of Rayleigh number (based on length of the channel) varying from $10^{10}$ to $10^{16}$, aspect ratio between 0.03 to 0.25 , the converging angle from 1 to $30^{\circ}$ and sloping angle from 0 to $60^{\circ}$. Finally, an application of this numerical correlation in two experimental prototypes is presented.
\end{abstract}

Keywords: Turbulent convective flows, inclined converging channels, numerical correlation.

*Corresponding author. Tel.: +34 968325 984; fax: +34 968325 999. E-mail: antonio.kaiser@upct.es 


\section{Notation}

$b \quad$ minimum inter-plate spacing in the channel (Fig. 1)

$c_{p} \quad$ specific heat at constant pressure

$g \quad$ gravitational acceleration

$\mathrm{Gr}_{l} \quad$ Grashof number, $g \beta\left(T_{w}-T_{\infty}\right) l^{3} / v^{2}$

$\mathrm{Gr}_{l} \quad$ Grashof number, $g \beta q l^{4} / \nu^{2} \kappa$

$h_{\chi} \quad$ local heat transfer coefficient along the wall, $\kappa(\partial T / \partial \eta)_{w} /\left(T_{w}-T_{\infty}\right)$

I turbulence intensity

$k \quad$ turbulent kinetic energy

$L \quad$ streamwise length of wall (Fig. 1)

$l \quad$ typical length, $l=L$ or $l=b$

$\mathrm{Nu}_{l} \quad$ average Nusselt number, Equation (21)

$\mathrm{Nu}_{L}, \mathrm{Nu}_{b}$ average Nusselt number based on $L$ and $b$, respectively

$\mathrm{Nu}_{l, \chi} \quad$ local Nusselt number, $h_{\chi} l / \kappa$, Equation (21)

$\mathrm{Nu}_{L, \chi}, \mathrm{Nu}_{b, \chi}$ local Nusselt number based on $L$ and $b$, respectively

$P \quad$ average reduced pressure

Pr Prandtl number, $\mu c_{p} / \kappa$

$q \quad$ wall heat flux

$\mathrm{Ra}_{l} \quad$ Rayleigh number, $\left(\mathrm{Gr}_{l}\right)(\mathrm{Pr})$

$\mathrm{Ra}_{L}, \mathrm{Ra}_{b} \quad$ Rayleigh number based on $L$ and $b$, respectively

$\mathrm{Ra}^{*} \quad$ modified Rayleigh number based on $b, \operatorname{Ra}_{b}(b / L)$

$\operatorname{Re}_{l} \quad$ Reynolds number, $U l / \mathrm{v}$

$T \quad$ average temperature

$T^{\prime} \quad$ turbulent temperature

$T_{w} \quad$ wall temperature

$T_{\infty} \quad$ ambient temperature

$U \quad$ typical velocity

$\bar{U} \quad$ average velocity at the inlet channel

$-\overline{T^{\prime} u_{j}} \quad$ average turbulent heat flux

$U_{j} \quad$ average components of velocity

$u_{j} \quad$ turbulent components of velocity

$-\overline{u_{i} u_{j}} \quad$ turbulent stress 


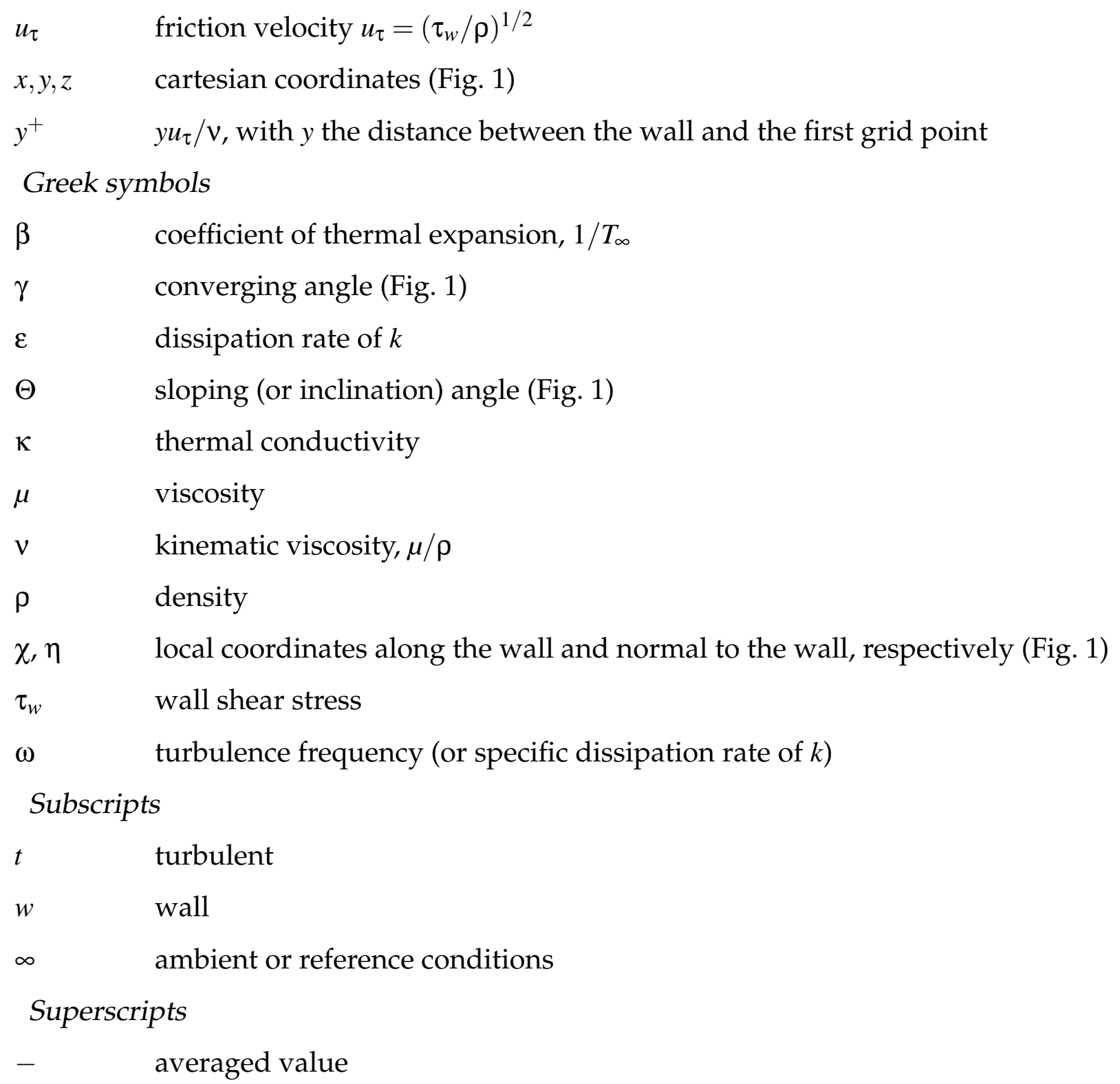




\section{Introduction}

The flows induced by natural convection appear in many engineering problems. Configurations formed by heated plates where these processes occur, have been the topic of intense study in past years. However, new systems with a different constructive disposition and applications in several fields (bioclimatic architecture, electronic cooling, nuclear energy cooling systems) are at the moment of great interest. Since typical applications of natural convection flows in smooth vertical channels are usually in small-scale devices (i.e. in electronic cooling equipment), most investigations were carried out for laminar flows. Frequently, flows are considered to be laminar at modified Rayleigh number $\mathrm{Ra}^{*} \leq 10^{5}$. As a consequence of the great scale of certain passive ventilation systems, as solar chimneys, Trombe walls, or roof collectors, the modified Rayleigh number is usually $\mathrm{Ra}^{*} \geq 10^{6}$, and the flow established becomes transitional or even fully turbulent. In this way, the study of problems such as the transition to turbulent regime or flows with walled-channel geometries including sloped and converging walls are key to assertively find out the real heating transmission existing in these new systems in a more realistic manner.

Specific heat transfer correlations for the whole applications range need to be provided to obtain a full understanding and design capacity of these kind of systems. Thus, the main objective became for the present research to obtain a generalized correlation for the average Nusselt number as a function of Rayleigh number, taking into account the effects of several geometrical parameters. This correlation could be employed in sizing methods of passive ventilation systems, for a given conditions. This paper describes a numerical investigation over the problem exposed. Special emphasis is made on the systematic comparisons of results obtained with experimental and numerical data from literature.

A large number of research published on laminar natural convection flow in channels exists; however, only few investigations have been reported on the turbulent case. Special attention has deserved transitional flows, as well as the using of different turbulence models and wall functions. Some relevant researches are exposed following. Lloyd and Sparrow [1] studied the conditions of transition to turbulence for a flow generated by natural convection on an isolated vertical plate and they established a value for the turbulence transition at Grashof number (based on the length of channel $L$ ) of $1.24 \times 10^{9}$. In [2] it is studied the same problem and they forecasted a critical value of local Grashof number of $1.17 \times 10^{6}$. Chen and Tzuoo [3] carried out an analysis of the simulation method of vortex instability and they ob- 
tained a critical value of the local Grashof number equal to $1.2 \times 10^{6}$, very closed to the one obtained in [2]. Tzuoo et al. [4] carried on with the existing studies regarding problems of natural convection on a vertical plate with turbulent regime improving the methods of turbulence measurements; they obtained a critical value of the local Grashof number of $6.2 \times 10^{6}$. Miyamoto et al. [5] have studied experimentally the flow generated by natural convection in turbulent regime in vertical channels where in one of the plates a constant heat flux is applied and the other plate is kept adiabatic. These authors established the transition for a local Grashof number in the order of $10^{13}$.

Numerically, turbulent two-transport equations models in combination with wall functions, have been usually applied in problems where forced convection boundary layers appear in order to calculate the velocity and temperature gradients close to a given wall. However, logarithmic wall functions do not seem to be appropriate in cases in which natural convection boundary layers take place. In this way, other authors [6] proposed new wall functions for numeric simulations of turbulent convective flows in vertical plates. In [7] it has been studied the scaling behavior of natural convection flows to propose wall functions for flows between two infinite differentially heated vertical walls. Based on a matching of inner and outer layer scaling relationships, an explicit expression for the mean profile in the matching region was presented. A range of Rayleigh number based on plate length of $5 \times 10^{5}-5 \times 10^{6}$ was considered by these authors. On the other hand, Henkes and Hoogendorn [8] studied turbulent convection in enclosures, stating the difficulties found in some turbulence models, to predict correctly the heat fluxes in these problems.

The latest tendencies seem to indicate the use of standard models, as $k-\varepsilon$, but for lowReynolds number, other alternative models such as $k-\omega$ for low-Reynolds number, and even the use of more complex models not based in the eddy viscosity. Hence, Peng and Davison [9] used the $k-\omega$ model to describe the turbulent flow generated by natural convection within a cavity. Xu et al. [10] studied the problem of turbulence generated by natural convection in a channel through a new model of turbulence which uses direct numerical simulation (DNS) in the region closed to the wall and also they utilized the $k-\varepsilon$ model for the region further away from the wall. In [11] it has been studied the same problems for modified Rayleigh numbers (based on width of the channel, $b$ ) between $10^{4}$ and $10^{6}$, and inclination angles of the channel with regards to the vertical, between $0^{\circ}$ and $90^{\circ}$, with a modified $k-\varepsilon$ turbulence model (Yang-Shih model) which takes into account the vanishing of turbulent kinetic energy 
close to the wall.

Fedorov and Viskanta [12] used the low-Reynolds number $k-\varepsilon$ turbulence model to calculate the induced flow and heat transfer in an asymmetrically heated, vertical parallel-plate channel. They compared their results with those obtained experimentally in [13]. They reported that the low-Reynolds number $k-\varepsilon$ model was capable of predicting the heat transfer in the system studied. They proposed that the turbulence intensity at the inlet of the channel affects to the location of the transition from laminar to turbulent flow as well as the average heat transfer. According to these authors, the flow becomes turbulent at a relative vertical distance $x / L$ measured from the channel inlet of 0.84 for a Rayleigh number based on the channel width of $10^{5}$, and 0.64 for a Rayleigh number of $10^{6}$.

From a different perspective, In [14] an experimental research of natural convection of air within a channel with similar performance conditions to those of Trombe walls is presented. Rodrigues et al. [15] investigated numerically a system formed by solar collectors (typified as open vertical channels by both ends) situated in the south face of a building as a passive way of improving inner air quality, without prejudice of comfort thermic requirements. They obtained results for different geometries and ambient conditions, and established that the induced flow was turbulent. Moreover, they carried out a two dimensional numeric simulation.

In view of these studies it can be observed that results of heat transfer for the transition and developed turbulent regime in channels are limited. They are referred in the majority of cases to asymmetrical heating and they do not contemplate on a systematically manner the influence of geometric parameters such as the converging angle, the inclination angle and the aspect ratio of the channel. Furthermore, some discrepancies in the location of the transition from laminar to turbulent regime exists. From a practical point of view, our aim consists of studying channels with roughly Trombe wall or solar chimney geometries. In the majority of cases, the flow established in this solar passive systems is transitional or fully turbulent, as pointed before. Hence this work is focused on the study of heat transfer processes by natural convection in convergent and inclined channels (described on Figure 1) in the laminar-turbulent transition and developed turbulent regimes with symmetrical isothermal heating.

The paper is organized as follows. First, the governing equations and turbulence model are introduced. Second, the numerical solution procedure for the full Navier-Stokes elliptic 
equations is also exposed. The flow has been assessed using the general-purpose Fluent and Phoenics codes, both based on a finite volume procedure. These codes have been validated enough to solve natural convective flows. Previously, satisfactory results for laminar studies have been obtained [16]. The working fluid is air, with a constant Prandtl number equal to 0.71. Later, in the section for discussion of results, validation of numerical results, influence of sloping and converging angles, and a generalized correlation for the average Nusselt number are explained. Finally, a checking of this numerical investigation in two experimental prototypes is presented.

\section{Mathematical model}

As it is well-known, in turbulent flows the instant variables included in the governing equations such as velocity, density, temperature and pressure fluctuate with time on an uncertain way, causing a strong mixing of transport quantities. As these fluctuations can be on a small scale and high frequency, it results in most cases, computationally very laborious to face the direct numerical simulation (DNS) of the problems outlined. However, some solutions have been put forward DNS for concrete problems [7, 17, 18].

Taking into account this plan, one of the most common practice in engineering applications consists in solving numerically the time-averaged governing equations, known as Reynolds-averaged equations. In the present study, the last approach is used. The closure problem generated in the conservation averaged equations is solved through the two transport equations $k-\omega$ model, as this does not imply an excessively high computational cost. Moreover, the $k-\omega$ model has some advantages in transitional flows. Indeed, this model allows to treat naturally the transition of laminar to turbulent regimes. No special models have been employed to trigger transition at certain location in the flow field; the transition point appears as the solution of the governing transport equations. Another advantage of the model is that it does not need to calculate wall distances for utilize in low-Re damping functions.

Since the temperature (and obviously, the pressure) variations are not too high, the Boussinesq approximation can be employed, assuming constants the thermophysical properties of the fluid, except for density variations in the buoyancy term in the vertical momentum equation. Applying the Boussinesq approximation, the simplified Reynolds-averaged steady two- 
dimensional governing equations are

$$
\begin{gathered}
\frac{\partial U_{j}}{\partial x_{j}}=0, \\
\left(\frac{\partial U_{i} U_{j}}{\partial x_{j}}\right)=-\frac{1}{\rho} \frac{\partial P}{\partial x_{i}}-g_{i} \beta\left(T-T_{\infty}\right)+\frac{\partial}{\partial x_{j}}\left(v \frac{\partial U_{i}}{\partial x_{j}}-\overline{u_{i} u_{j}}\right), \\
\frac{\partial\left(T U_{j}\right)}{\partial x_{j}}=\frac{\partial}{\partial x_{j}}\left(\frac{v}{(\operatorname{Pr})} \frac{\partial T}{\partial x_{j}}-\overline{T^{\prime} u_{j}}\right),
\end{gathered}
$$

where $U$ and $T$ are the average velocity and average temperature, respectively; $P$ the average reduced pressure, difference between the pressure and the ambient pressure $P_{\infty}$, given by the hydrostatic law $\left(\mathrm{d} P_{\infty} / \mathrm{d} x=-g \rho_{\infty}\right) ; \beta$ is the thermal expansion coefficient, equal to $1 / T_{\infty}$ for a perfect gas. The turbulent stress $-\overline{u_{i} u_{j}}$ and turbulent heat flux $-\overline{T^{\prime} u_{j}}$ are provided from the turbulence closure model, which are explained below, assuming that

$$
-\overline{u_{i} u_{j}}=2 v_{t} S_{i j}-\frac{2}{3} k \delta_{i j}, \text { and }-\overline{T^{\prime} u_{j}}=\frac{v_{t}}{\operatorname{Pr}_{t}} \frac{\partial T}{\partial x_{j}},
$$

being $v_{t}$ and $\operatorname{Pr}_{t}$ the turbulent kinematic viscosity and turbulent Prandtl number, respectively; $S_{i j}$ is the mean strain tensor, $S_{i j}=\left[\left(\partial U_{i} / \partial x_{j}\right)+\left(\partial U_{j} / \partial x_{i}\right)\right] / 2, \delta_{i j}$ the Krönecker delta, and $k$ de kinetic turbulent energy, given by

$$
k=\frac{\overline{u_{x}^{2}}+\overline{u_{y}^{2}}+\overline{u_{z}^{2}}}{2} .
$$

\subsection{Turbulence model}

The first turbulence model of two transport equations was the $k-\omega$ model, proposed originally in [19]. This model presents equations for the transport of kinetic turbulent energy, $k$, and the transport of dissipation per unit turbulent kinetic energy, $\omega$. The $k-\omega$ model proposed by Wilcox [20] has been used in the present study mainly because it includes a lowReynolds number extension for near-wall turbulence and allows the solving of transitional flows. As pointed before, the model does not imply an excessively high computational cost; for example, it does not require the calculation of wall distance or damping functions based on the friction velocity. The $k-\omega$ transport equations model is presented below:

1. Transport equation for kinetic turbulent energy $k$

$$
\frac{\partial k}{\partial t}+U_{j} \frac{\partial k}{\partial x_{j}}=\frac{\partial}{\partial x_{j}}\left[\left(v+\frac{v_{t}}{\sigma_{k}}\right) \frac{\partial k}{\partial x_{j}}\right]+\overline{u_{i} u_{j}} \frac{\partial U_{i}}{\partial x_{j}}-\beta^{*} f_{\beta^{*}} k \omega .
$$


- In the diffusion term, the effective diffusivity is $v+\left(v_{t} / \sigma_{k}\right)$, and the kinematic eddy viscosity is given by

$$
v_{t}=\alpha^{*} \frac{k}{\omega}
$$

being $\alpha^{*}$ a function that damps the turbulent viscosity causing a low-Reynolds number correction, given by

$$
\alpha^{*}=\alpha_{\infty}^{*}\left(\frac{\alpha_{0}^{*}+\operatorname{Re}_{t} / R_{k}}{1+\operatorname{Re}_{t} / R_{k}}\right), \text { with } \operatorname{Re}_{t}=\frac{k}{v \omega} .
$$

- The turbulence production term is $\overline{u_{i} u_{j}}\left(\partial U_{i} / \partial x_{j}\right)$.

- In the turbulence dissipation term, $-\left(\beta^{*} f_{\beta^{*}} k \omega\right)$, the factor $f_{\beta^{*}}$ is

$$
f_{\beta^{*}}=\left\{\begin{array}{ll}
1, & \xi_{k} \leq 0 \\
\frac{1+680 \xi_{k}^{2}}{1+400 \xi_{k}^{2}}, & \xi_{k}>0
\end{array}, \text { with } \xi_{k} \equiv \frac{1}{\omega^{3}} \frac{\partial k}{\partial x_{j}} \frac{\partial \omega}{\partial x_{j}},\right.
$$

and $\beta^{*}$ is a damping function of $\operatorname{Re}_{t}$,

$$
\beta^{*}=\beta_{\infty}^{*}\left(\frac{4 / 15+\left(\operatorname{Re}_{t} / R_{\beta}\right)^{4}}{1+\left(\operatorname{Re}_{t} / R_{\beta}\right)^{4}}\right) .
$$

The optative additional production of turbulent buoyancy, $-g_{i} \beta\left(v_{t} / \operatorname{Pr}_{t}\right)\left(\partial T / \partial x_{i}\right)$, have been neglected, since no stratification temperature fields is expected in the present work.

2. Transport equation for specific dissipation rate or turbulence frequency $\omega$

$$
\frac{\partial \omega}{\partial t}+U_{j} \frac{\partial \omega}{\partial x_{j}}=\frac{\partial}{\partial x_{j}}\left[\left(v+\frac{v_{t}}{\sigma_{\omega}}\right) \frac{\partial \omega}{\partial x_{j}}\right]+\overline{u_{i} u_{j}} \frac{\partial U_{i}}{\partial x_{j}} \alpha \frac{\omega}{k}-\beta f_{\beta} \omega^{2} .
$$

- In the production term, $\overline{u_{i} u_{j}}\left(\partial U_{i} / \partial x_{j}\right) \alpha(\omega / k), \alpha$ is another damping function,

$$
\alpha=\frac{\alpha_{\infty}}{\alpha^{*}}\left(\frac{\alpha_{0}+\operatorname{Re}_{t} / R_{\omega}}{1+\operatorname{Re}_{t} / R_{\omega}}\right) .
$$

- In the dissipation term $-\left(\beta f_{\beta} \omega^{2}\right), f_{\beta}$ is:

$$
f_{\beta}=\frac{1+70 \xi_{\omega}}{1+80 \xi_{\omega}}, \text { with } \xi_{\omega} \equiv\left|\frac{\Omega_{i j} \Omega_{j k} S_{k i}}{\left(\beta_{\infty}^{*} \omega\right)^{3}}\right|,
$$

being $\Omega_{i j}$ and $S_{k i}$ the mean-rotation and mean-strain tensors, respectively defined by

$$
\Omega_{i j}=\frac{1}{2}\left(\frac{\partial U_{i}}{\partial x_{j}}-\frac{\partial U_{j}}{\partial x_{i}}\right), \quad S_{i j}=\frac{1}{2}\left(\frac{\partial U_{i}}{\partial x_{j}}+\frac{\partial U_{j}}{\partial x_{i}}\right) .
$$

(The factor $\xi_{\omega}$ is zero for two-dimensional flows, and so, $f_{\beta}=1$.) 
Obviously, since the flow is steady, $\partial k / \partial t=\partial \omega / \partial t=0$. The following experimental constants complete the model, see Wilcox [20] and Fluent manuals [21]: $\sigma_{k}=2.0, R_{k}=6.0, \alpha_{0}^{*}=$ $\beta_{i} / 3, \alpha_{\infty}^{*}=1, \beta_{i}=0.072, R_{\beta}=8.0, \beta_{\infty}^{*}=0.09, \sigma_{\omega}=2.0, R_{\omega}=2.95, \alpha_{\infty}=0.52, \alpha_{0}=1 / 9, \beta=\beta_{i} . \mathrm{A}$ constant value of $\operatorname{Pr}_{t}=0.86$ is used in the computations.

With the above formulation, it can be obtained that dissipation rate of $k$ is $\varepsilon=\beta^{*} \omega k$. The functions $f_{\beta^{*}}$ and $f_{\beta}$ and the values of $\alpha$ and $\beta_{o}$ have been calibrated to provide spreading rates that are consistent with measurements for far wakes, mixing layers and plane, round and radial jets. These modifications eliminate confusion regarding the $k-\omega$ model's sensitivity to freestream boundary conditions [20]. Its effect is undervalued in the proximity of the wall, mainly because $\omega$ is generally very big next to the solid walls and therefore, as it can be deduced from algebraic expressions, the value of functions $f_{\beta^{*}}$ and $f_{\beta}$ are close to 1 . Thus, this dissipation coefficients have a very small effect on boundary-layers flows, such as the problem in study. The dumping functions $\alpha, \alpha^{*}$ and $\beta^{*}$ are set to unity in the core turbulent region, where $\operatorname{Re}_{t}>>1$.

\subsubsection{Laminar-turbulent transition}

One of the main objectives in the development of turbulence models in the last years is to determine the transition from laminar to turbulent regime. The $k-\omega$ model used in the present study allows us to determine descriptively the transition point. In order to understand the way this model solves the problem of transition it is necessary to group in the equations of $k$ and $\omega$ the dissipation and production terms [20]:

$$
\overline{u_{i} u_{j}} \frac{\partial U_{i}}{\partial x_{j}}-\beta^{*} f_{\beta^{*}} k \omega=P_{k} \beta^{*} \omega k, \quad \overline{u_{i} u_{j}} \frac{\partial U_{i}}{\partial x_{j}} \alpha \frac{\omega}{k}-\beta f_{\beta} \omega^{2}=P_{\omega} \beta^{*} \omega^{2},
$$

where the net specific production per unit-dissipation terms in both equations, $P_{k} \mathrm{y} P_{\omega}$ are respectively defined as:

$$
P_{k}=\frac{\alpha^{*}}{\beta^{*}}\left(\frac{\partial U / \partial y}{\omega}\right)^{2}-1, \quad P_{\omega}=\frac{\alpha \alpha^{*}}{\beta}\left(\frac{\partial U / \partial y}{\omega}\right)^{2}-1 .
$$

With regards to the previous statements, it can be deduced that if the turbulent kinetic energy is zero, the eddy viscosity vanishes and the $\omega$ equation uncouples from the $k$-equation. Consequently, the $k-\omega$ model has a nontrivial laminar-flow solution, with $v_{t}=0$ for $\omega$-equation. On the other hand, the signs of $P_{k}$ and $P_{\omega}$ determine whether $k$ and $\omega$ are amplified or reduced in magnitude. Since it is not clear how these signs influence over transition, Wilcox 
[20] carried out a study applying the Blausius transformation for a laminar boundary layer, and shown the relation between Reynolds number and the sign of such terms, which determines the amplification or reduction of $P_{k}$ and $P_{\omega}$ and, therefore, the enhancing or damping of turbulence.

\subsubsection{Near-wall settings}

The non-slip boundary condition imposed at the walls modifies the value of the average velocity of flow and the viscous damping reduces the turbulent velocity fluctuations. However, away from this viscous sub-layer, turbulence increases quickly by the production of turbulent kinetic energy caused by the average velocity gradients. The zone close to the wall can be considered as a main source of average vorticity and turbulence. We can therefore mention that a precise representation of flow in the near-wall region will determine the success of flow predictions in their presence. This is the approach adopted in the present study.

According to the universal behavior near the wall (usually, a logarithmic law), some turbulence models impose in this region the so-called wall functions which simulate this behavior, avoiding having to solve the flow there. This could be appropriate in flows fully developed. However, in transitional flows, the turbulence production does not have to balance itself with the turbulence dissipation near the walls, and the hypothesis of universality of the boundary layer profiles (the law of the wall) is not fulfilled. The $k-\omega$ model allows to work with wall functions if mesh is sufficiently coarse near the wall, with $y^{+}>30$, or otherwise, to solve the flow within viscous sub-layer if the mesh is sufficiently fine and presents nodes inside the viscous sub-layer, with non-dimensional sub-layer scaled distance $y^{+} \approx 1$. At least 10 grid lines should be included in the viscous sub-layer [6].

\subsection{Boundary conditions}

The boundary conditions have been imposed for both Fluent and Phoenics codes in a similar way. At the inlet section, a total average reduced pressure equal to $P_{T}=P+\rho\left(U_{x}^{2}+U_{y}^{2}\right) / 2=0$ is imposed, and the temperature is fixed equal to the ambient temperature $T_{\infty}$. The initial values for turbulent kinetic energy $k$ and turbulence frequency $\omega$ have been imposed by a turbulence intensity equal to $2 \%$ in most outlined cases. This parameter is usually defined as follows:

$$
I=\frac{[(2 / 3) k]^{1 / 2}}{\bar{U}},
$$


where $\bar{U}$ is the average velocity at the channel inlet. It is calculated from the flow field solution, for each iteration until the converged solution is obtained, by a transversal integration of streamwise velocity field, at the channel inlet. Thus, at inlet,

$$
k_{\text {init }}=\frac{3}{2} I^{2} \bar{U}^{2}, \quad \omega_{\text {init }}=\frac{k_{\text {init }}}{v_{t}} .
$$

Under certain conditions, conduction effects at inlet could be important. It depends on $\mathrm{Ra}^{*}$, the aspect ratio and the domain considered. Nevertheless, as an example, the conduction effects begin to be significant for Ra* lower than 1, for a certain geometry of the extended domain [22]. In these cases, an extended computational domain at inlet is required. For laminar flows, the effect of including an entrance region to the domain is considered in [22]. In present work, for mainly high-Rayleigh flows, the effects of including that additional domain at the channel inlet have been obtained negligible. Thus, the diffusion effects at the inlet channel have been omitted.

At the outlet section, it is considered that the average reduced pressure is $P=0$. It is clear that since an initial value of velocity at inlet section is not known a priori in natural convection flows, a reference level for pressure is necessary for the numerical simulation of the elliptic problem. Physically, at the exit, the flow discharges in a jet-like manner to the ambient without pressure recovery. Consequently, the streamwise variations of velocity components, temperature, turbulent kinetic energy and turbulence frequency are neglected.

At the walls, the no-slip boundary conditions is imposed on the average and turbulent velocity components. In the isothermal cases, it has been considered a fixed temperature with a value of $T=T_{w}$; at the walls with a fixed heat flux $q$, it has been imposed $\kappa(\partial T / \partial y)=q$, with $y$ the cross coordinate, and at the adiabatic walls, $\partial T / \partial y=0$.

The boundary conditions for $k$ and $\omega$ must be consistent with the equations for the lowReynolds number $k-\omega$ turbulence model, imposing $k=0$. For high-Reynolds problems, the wall functions for $k$ and $\omega$ are:

$$
k=\frac{u_{\tau}^{2}}{\left(\beta_{\infty}^{*}\right)^{1 / 2}}, \text { and } \omega=\frac{k^{1 / 2}}{\left(\beta_{\infty}^{*}\right)^{1 / 4} \kappa_{v} y}, \quad \text { as } y^{+} \rightarrow \infty,
$$

with $\beta_{\infty}^{*}=0.09$ and $\kappa_{v}$ the von Kármán constant, equal to 0.41 . While, for low-Reynolds behavior, with $y^{+}$low enough, $k$ must vanish and, by means of a balance for the $w$-equation, an asymptotic expression for $\omega$ as the wall is approached [20],

$$
k=0, \text { and } \omega=\frac{K v}{\beta_{\infty}^{*} y^{2}},
$$


being the theoretical value for $K$ equal to 2. The value for $\omega$ given by Equation (20) is employed for calculating $\omega$ at the computational first point close to the wall surface.

\section{Numerical computations}

Results presented in this work have been obtained by using both the general-purpose Fluent and Phoenics codes, based on a finite volume procedure. The convergence criterion in each case was $\left(\phi^{i+1}-\phi^{i}\right) / \phi^{i} \leq 10^{-5}$, where $i$ denotes iteration number and $\phi$ can stand for any of the dependent variables. In addition, the normalized residuals for mass, momentum, energy and turbulent variables, for the fully flow field, are required to be below $10^{-3}$ in order to reach a fully converged solution. The combination of both criterions provides an effective overall convergence procedure. In Fluent, the equations are discretised on a staggered grid, using the 'Presto' scheme, which is similar to the staggered-grid scheme with structured meshes employed in Phoenics, with a second-order scheme for the convective terms. In Fluent, the Simple algorithm [23] is employed to solve the coupling between continuity and momentum equations through pressure (in Phoenics, the Simplest algorithm, which is a modified version of the Simple algorithm, is employed). In Phoenics, the results have been achieved employing a second-order differential scheme of 'Muscl' type for the convective terms [24]. In this research, other alternative differential schemes, as 'Quick', have been tested, but nearly identical results have been obtained.

In Fluent and Phoenics codes, the $k-\omega$ turbulence model described previously has been implemented. The wall damping functions employed in both codes are practically coincident. Normally, convergence is proving difficult with low-Re turbulence models. In this work, the $k-\omega$ model have demonstrated to be stable and convergent enough, for the wide range of Rayleigh number studied.

A structured, non-uniform mesh has been built in both codes. In order to ensure the accuracy of the numerical results, a grid dependence study was performed (see Table 1). For both the horizontal and vertical direction, and for each grid and aspect ratio, a power-law distribution was used to obtain a fine grid near walls, inlet and outlet sections of the channel. Different ratios between number of cells in the vertical and horizontal directions were tested for each case solved. The reference for this sensibility study was the case of a convergent channel with converging angle $\gamma=12^{\circ}$, inclination angle $\Theta=0^{\circ}$, aspect ratio $b / L=0.1$ and 
$\mathrm{Ra}^{*}=10^{9}$. Once the optimum mesh is determined, this was extended to the rest of cases. Table 1 shows the values of average Nusselt number and the error committed taking as a reference the value which achieves not being affected by the size of the mesh, with regards to the number of cells of the considered domain and the value of $y^{+}$. Even though these last two parameters affect clearly the values of the Nusselt number, $y^{+}$seems to be key to determine the optimum mesh.

The results obtained with Fluent and Phoenics show that meshes with $y^{+}$less than unity give errors less than 3\%. Therefore, in view of these results the criterion considered to achieve mesh independence in the other cases studied was to consider as valid those cases with a $y^{+}$ less than this value, preferably in the range $0.1 \leq y^{+} \leq 0.5$. This fact can be observed in Figure 2 , showing that the average Nusselt number nearly reaches a constant value for $y^{+}$less than unity. The reason for such a drastic independence change is because below this $y^{+}$the flow through boundary laminar sub-layer is calculated without wall functions, obtaining a more detailed and precise simulation of the flow behavior within this region. As pointed above, fineness of grids in wall zones was obtained by means of power-law cell size distributions. Most cases were computed with a $120 \times 80$ grid, including at least 10 grid points in the viscous sub-layers. In this way, since $\omega$ values reached at the first node close to the wall are usually very high, strong relaxation devices for computing variables was used to get enough accurate solutions in the turbulent cases.

\section{Discussion of results}

Before presenting the numerical results obtained for heat transfer in channels with symmetrical heating, a comparison with the results proposed in $[7,15,5,25,11]$ has been carried out. In addition, special care deserved the transition point to turbulence.

\subsection{Validation and turbulence starting point}

Versteegh and Nieuwstadt [7] studied the flow established by natural convection within a vertical channel with a temperature difference between walls of $39.1^{\circ} \mathrm{C}$, enough to reach the turbulent regime with the geometric and physical conditions given, as pointed these authors. The authors address the problem from a numerical viewpoint. In the simulation they used a 3D model with direct numerical simulation (DNS), imposing in the inlet and outlet a con- 
dition of periodicity. This way they avoided resolving the transition from laminar regime to turbulent regime. Aiming to validate their numeric results, the authors presented experimental results of some of the analyzed cases. Figure 3 shows the profiles of nondimensional temperature obtained with DNS and experimentally [7], and also the profiles given by the 2D simulation with the $k-\omega$ model by means of Fluent and Phoenics codes. The results obtained through these codes do not show significant differences. It can be observed a good agreement between our results and those obtained with DNS, with discrepancies with respect to experimental results less than $5 \%$ in most cases. Given the condition of periodicity, the Rayleigh number was based in the width of the channel $\left(\mathrm{Ra}_{b}=g \beta\left(T_{w}-T_{\infty}\right) b^{3} / \alpha v\right)$. Grids employed in [7] varied from $(180 \times 90 \times 48)$ to $(432 \times 216 \times 96)$ cells.

Rodrigues et al. [15] analyzed the turbulent flow generated by natural convection in a vertical channel. They solved numerically the averaged Reynolds equations, using Boussinesq closure model for the Reynolds-stress tensor, where the value of turbulent viscosity is obtained from the $k-\varepsilon$ turbulence model. Given that the outlined standard $k-\varepsilon$ model is only applicable for high-Reynolds number, wall functions have been used near the wall. A very important research of this study, consists of the way in which the authors treat the problem of transition from laminar to turbulent regime. Based on [26] and [27], they fixed the transition at local Grashof number equal to $10^{12}$, under Neumann conditions of uniform wall heat fluxes. In this way, the same transition point was reached, for isolated flat plate, and for channel formed by two plates. According to this critical value, the authors proposed two computational domains, one where the local Grashof number is less than this value, resolving the laminar equations, and other domain where the local Grashof number is higher, applying then the turbulence model previously cited. They considered asymmetric heating conditions, imposing a constant heat flux at the walls, as pointed above, to simulate heating conditions from a solar-air collector. In Figure 4 it can be observed that for the results obtained in this work, from a certain value of the streamwise co-ordinate, the wall temperature starts smoothly to decrease. This is caused by a mixing between the different fluid layers, or in other words, by the transition from laminar to turbulent regime. These results established a transition near to a critical local Grashof number of $0.9 \times 10^{12}$ for Phoenics and $2.5 \times 10^{12}$ for Fluent, near to the predicted value proposed in [26] and [27], and fixed in [15].

Focusing on transition, for high enough Rayleigh (or equivalent, Grashof) numbers, it can be observed an initial zone where the flow yields laminar, an intermediate zone of transition 
and a final region where the flow become fully turbulent. The flow is initiated with a tiny turbulent kinetic energy. As the flow near the wall starts developing, the production terms of turbulent kinetic energy (such as the shear rate production generated in the wall) overcome the average of dissipation of this energy, resulting in the transition to turbulence. In the transversal direction to the wall, into the zone where the turbulence regime has been reached, it can be observed the same approach than in [10]. It can be distinguished, very close to the wall (within the boundary layer) a zone where the viscous diffusion of the shear rate is totally balanced by the dissipation, and a laminar behavior is obtained. Further from the wall (still within the boundary layer) the shear rate alongside the thermal gradients overcome the dissipation effects given place to a turbulent pattern, which diffuses itself further away from the boundary layer. Some authors, who have studied the turbulent boundary layer generated by natural convection [17] and [18], detected through direct numerical simulation, close to the wall, a negative production caused by the shear rate in the equation of turbulent kinetic energy. This negative production has been registered with a stratified field of temperature. This singular behavior has not been detected through the $k-\omega$ model in the present study.

Other authors have centered their research on the problem of laminar to turbulent transition in channels and slots but for asymmetrical heating. So, some differences with respect to symmetric heating conditions in channels may be appreciated. Habib et al. [25] studied experimentally the problem of turbulent heat transfer in channels with plates heated asymmetrically at uniform temperature. They presented results of velocity profiles in different sections of the channel and compared these results with those proposed in [13]. They defined the Rayleigh number as a function of the channel length. Some of their results are presented in Figure 5. In [25] it was concluded that the flow was turbulent for $\operatorname{Ra}_{L} \geq 10^{5}$, as Boudjemadi et al. [28] proposed. However, these authors centered their studies in vertical slots, not in open channels. Fedorov and Viskanta [12] detected the transition to turbulent regime by the dramatic increase of the local Nusselt number for a given streamwise coordinate, for values of local Rayleigh number based on channel inter-plate spacing in the order of $10^{5}-10^{6}$. This corresponds with a local Rayleigh number based on channel length in the order of $10^{11}$, taking into account the channel aspect ratio considered. It should be noticed that for an isolated isothermal plate, some authors $[29,30]$ point out that the transition occurs for a local Grashof number close to $10^{9}$.

In view of the results obtained, the $k-\omega$ model for low-Reynolds numbers seems to sim- 
ulate correctly the turbulence established by natural convection, adjusting to the available experimental results with a reasonable agreement. For the considered problem, it is reached that the lack of $f_{\beta^{*}}$ and $f_{\beta}$ functions in the Phoenics turbulence model does not affect the results obtained. Once the $k-\omega$ model is validated, the results obtained with such a model for the heat transfer in vertical channels varying the Rayleigh number based on $L$ from $10^{6}$ to $10^{16}$ will be reported. Average Nusselt number has been calculated as follow:

$$
\mathrm{Nu}_{l}=\frac{1}{L} \int_{0}^{L} \mathrm{Nu}_{l, \chi} \mathrm{d} \chi, \text { with } \mathrm{Nu}_{l, \chi}=h_{\chi} l / \kappa
$$

where $h_{\chi}$ is the local heat transfer coefficient and $l$ a typical length.

Another parameter to be considered when determining the transition point from laminar to turbulent regime is the turbulence intensity at the channel inlet [12]. Figures 6 and 7 show the influence exerted by the turbulence intensity in the local heat transfer at the inlet of the channel. In these figures, it is presented the local Nusselt number based on $b, \mathrm{Nu}_{b, x}$ at one of the walls for a modified Rayleigh number (based on $b$ ) of $1.6 \times 10^{6}$ and $1.6 \times 10^{8}$, respectively, and different turbulence intensities (2\%,5\% 10\% and 20\%). The results show that the bigger the turbulence intensity at the inlet, the bigger the flow mixing in the inlet zone and the local transition between laminar and turbulent regime is less brusque (Figure 7). As expected, turbulence transition may occur for low local Grashof (or Rayleigh) number, if a great turbulence level it is imposed at the inlet of the channel [12]. These differences leads to obtain higher average Nusselt number. A maximum deviation for Nusselt number close to $18 \%$ between one case with $I=20 \%$ and other with $I=2 \%$, for a transitional Rayleigh number of $\mathrm{Ra}^{*}=1.6 \times 10^{6}$ (equal to $\mathrm{Ra}_{L}=10^{9}$ ), have been reached (Figure 6). These differences are significant in a small range of the Rayleigh number $\left(\operatorname{Ra}_{L}=10^{8}-10^{10}\right)$ within the set of the laminar-turbulent transition. For $\mathrm{Ra}^{*}=1.6 \times 10^{8}$ (equal to $\mathrm{Ra}_{L}=10^{11}$ ), maximum deviation is only equal to $4 \%$. In fully turbulent flow these differences decrease dramatically, and become negligible. Transition effects start to be detected from values of $\mathrm{Ra}_{L}$ higher than $10^{8}$. At this time it can be observed a light increase of the local heat transfer as the flow starts developing on the wall, after reaching a certain minimum, passing from a descendent trend to an ascent (see Figure 7). This effect is more visible in the curve of $I=0.02$.

As a distinctive feature of thermal passive systems, no external mechanical devices are employed. So, a low value of turbulence intensity must be considered, if additional turbulence generating does not involved. In this way, a low value of initial turbulence at inlet $I=2 \%$ has been imposed in most cases. With the commonly used value of $I=2 \%$, a mean- 
ingful physical flow it is maintained.

The results obtained for the average Nusselt number based on the length of the channel $L$ as a function of Rayleigh number also based in $L$ for different aspect ratios is presented in Figure 8. It can be appreciated that in fully turbulent regimes, the results obtained for different aspect ratios are overlapped. However, in the wide transition range, for $\mathrm{Ra}_{L}$ which are comprised between $10^{8}$ and $10^{11}$, it does not seem so clear, as we can observe a light influence of the aspect ratio in the heat transfer. On the other hand, in this figure it can be appreciated a change of trend in the results of $\mathrm{Nu}_{L}$ for a $\mathrm{Ra}_{L}$ of the order of $10^{9}$, agreeing with [29] which summarize the transition from laminar to turbulent regime for vertical plates in $\operatorname{Ra}_{L} \approx 10^{9}$. Thus, according with above papers analyzed, the roughly limit $10^{9}$ can be accepted as good for transition point from laminar to turbulent flow, for isolated plate and for channels with high aspect ratio, taking into account some light deviations caused by geometrical parameters (as sloping angles) and initial turbulence intensity considered.

In this work, using Phoenics code, with a low level of initial turbulence intensity $(I=2 \%)$ and an aspect ratio of $b / L=0.5$, the transition takes place at $\operatorname{Ra}_{L} \cong 10^{9}$. For $b / L=0.03$, transition occur for $\mathrm{Ra}_{L} \cong 10^{11}$, while Fedorov and Viskanta [12] obtained turbulent flow for $\mathrm{Gr}_{b}=10^{6}$ and $b / L=0.0125$, i.e. for $\mathrm{Ra}_{L}=3.6 \times 10^{11}$, with $I=0$. In this study, for intermediate values of $b / L(0.1,0.2$ and 0.25$)$, the transition happened in the range $5.0-8.0 \times 10^{9}$.

The numerical results obtained with Fluent and Phoenics present variations around 5\%, with an identical trend for different Rayleigh number. In Figure 8 it can be observed that for values of $\mathrm{Ra}_{L}$ higher than $10^{12}$, there is a detachment of the results obtained for $\mathrm{Nu}_{L}$ with regards to vertical isolated plate correlation proposed in [30], $\mathrm{Nu}_{L}=0.1(\mathrm{Ra})^{1 / 3}$. Furthermore, the results obtained for the average Nusselt number in the isothermal channel for high aspect ratios (as pointed above, $b / L=0.5$ is the highest aspect ratio for which results have been obtained) do not match with those proposed in [29] for isolated plate, $\mathrm{Nu}_{L}=$ $\left(0.825+0.387 \mathrm{Ra}_{L}^{1 / 6}\right)^{2}$. The relationship between the Nusselt and Rayleigh numbers $\mathrm{Nu}_{L} \sim$ $\mathrm{Ra}_{L}^{1 / 3}$, proposed by these authors and by others from the literature, seems lightly different with respect to the correlation proposed in this study for channel configuration, where the exponent is 0.37 , as it is stated in the following paragraphs. 


\subsection{Influence of sloping angle}

In this paragraph, the influence of the sloping angle of the centerline line of the channel on heat transfer will be described. For laminar regime, Azevedo and Sparrow [31] have detected experimentally the existence of a secondary flow in a sloped channel for a certain angle (less than $45^{\circ}$ ). This secondary flow, produced because the buoyancy effects close to the wall forces the flow to detach itself from the wall, allowing a free region where the cold air, which is located in the upper zone, remains. This effect results in important variations in the Nusselt number when a heat flux is imposed at the lower plate and the upper one is remained adiabatic. In the case of symmetrical heating, even though secondary flow is produced, the average Nusselt number is hardly affected. In turbulent regime, this secondary flow has been observed and studied in [32] for the case of a sloping plate, proving that its presence results in an advance of the transition from laminar to turbulent regime, and therefore an improvement in the average Nusselt number. In the cases studied in this research, secondary flow in sloped channels has not been detected. These authors, among others, propose the inclusion of flow perturbations to reproduce this behavior. In the results obtained through this study, it can be observed that as the sloping angle increases, the turbulence intensity generated in the lower plate is higher to that generated in the upper plate. It can also be appreciated an asymmetrical field of velocity in the channel exit.

Said et al. [11] studied numerically the problem of heat transfer in this configuration employing a low-Reynolds $k-\varepsilon$ model, for a wide range of sloping angles $\left(0 \leq \Theta \leq 90^{\circ}\right)$, even though for a very reduced range of the modified Rayleigh number $\left(10^{4} \leq \mathrm{Ra}^{*} \leq 10^{6}\right)$, in the laminar to turbulent regime range. In fact, it is possible to find laminar numerical solutions in this range of $\mathrm{Ra}^{*}$. These authors proposed that the influence of sloping angle can be corrected applying to the modified Rayleigh number the factor $(\cos \Theta)^{0.5}$. In present research, results of the heat transfer for a range of inclination angle of $0 \leq \Theta \leq 60^{\circ}$ and modified Rayleigh number of $10^{4} \leq \mathrm{Ra}^{*} \leq 10^{12}$, have been presented. Figure 9 shows a comparison between the results obtained in [11] and the results of this study. For the cases studied in this research, the influence of sloping angle is achieved including the factor $(\cos \Theta)$ in the definition of the Rayleigh number. Said et al. [11] proposed the factor $(\cos \Theta)^{0.5}$; it should be noticed that these authors have extended the study to sloping angles of $90^{\circ}$. The use of Rayleigh number based on $(\cos \Theta)$ factor represents the assumption of an already widely employed practice for inclined plates. In a converging or sloped channel, the buoyancy force acts not only in 
the streamwise direction, since a component also exists perpendicular to the heated wall. Thus, the specific buoyancy force that accelerates the fluid in the thermal boundary layer is $\left[g \beta\left(T-T_{\infty}\right) \cos \Theta\right]$ instead of $\left[g \beta\left(T-T_{\infty}\right)\right]$. Later on, a correlation for the case of fully turbulent flow, which includes both the sloping angle $(\Theta)$ and the convergence angle of the plates $(\gamma)$, will be exposed.

As it is stated in the following paragraphs, the heat transfer on channel plates in turbulent regime is slightly influenced by the aspect ratio. In Figure 9 it is also included the laminar correlation proposed in [16] for the average Nusselt number in a channel with isothermal heated plates, for symmetric heating conditions, Ra* varying from 1 to $10^{6}$ and $\gamma$ from $0^{\circ}$ to $30^{\circ}$. This correlation has been redefined with regards to the Rayleigh and Nusselt number based on $L$, to be included in Figure 9.

\subsection{Influence of converging angle}

For convergent sloped channels, best correlation of results obtained was reached by using the minimum inter-plate spacing to describe the problem studied [16]. Qualitatively it can be observed that for a same Rayleigh number (within the range of turbulent flow), when the convergence angle of plates increasing, there is an increase in turbulent kinetic energy in the core of the channel. This redistribution of turbulent kinetic energy gives place to an advance in the transition from laminar to turbulent regime within the boundary layer, as it can be observed in Figure 10. This advance in the transition does not result in a substantial increase in the net heat transfer, as when the convergence angle is increased the buoyancy effects in the region near the wall is reduced. Particularly, the difference in the average Nusselt number between the cases of $\gamma=0^{\circ}$ and $\gamma=12^{\circ}$, for $\operatorname{Ra}_{L}=10^{12}$ and an aspect ratio of 0.1 , is of $2.5 \%$. The most important factor to be considered in order to evaluate the influence of the convergence angle is the reduction in buoyancy effects caused by the slope of the walls. Once again it is proved that the influence in this parameter is corrected by introducing the $(\cos \gamma)$ factor in the Rayleigh number expression. These results can be correlated through the following correlation (Figure 11)

$$
\mathrm{Nu}_{L}=C\left(\operatorname{Ra}_{L} \cos \gamma\right)^{m}
$$

with $C=0.0354$ and $m=0.371$. This expression is also applicable to the previous case, for vertical channels with parallel plates in which $\gamma=0^{\circ}$. 


\subsection{Influence of sloping and converging angles}

To obtain the influence of the sloping angle on the heat transfer in convergent channels for turbulent regime, the cases presented have been solved for converging angles of $\gamma=15^{\circ}$ and $30^{\circ}$ and for different sloping angles of the channel with regards to the vertical: $\Theta=15^{\circ}, 30^{\circ}$, $45^{\circ}$ and $60^{\circ}$.

Figure 12 shows the distribution of non-dimensional turbulent kinetic energy, as well as velocity fields for $\mathrm{Ra}^{*}=10^{8}, \gamma=30^{\circ}$ and sloping angles of $0^{\circ}$ and $60^{\circ}$. It can be observed that the variation of the sloping angle produces significant changes not only in flow pattern but also in the distribution of turbulent kinetic energy. However, these variations do not lead to significant changes in the average Nusselt number, for an equal value of $\mathrm{Ra}_{L}$, once corrected with the factors $(\cos \gamma)(\cos \Theta)$. In the first case, when the channel is sloped, there is an increase of the turbulent kinetic energy next to the lower wall and, as a consequence, a higher heat transfer at wall. This increase compensates the diminution of the heat transfer in the upper wall. In this way, the net variations of a non sloped configuration with regards to a sloped one are mainly caused by the reduction of buoyancy forces on the core of fluid placed in the channel, as it happened in the laminar regime [16]. Once again, the cosine correction is presented as a necessary factor to achieve an overlap of results (Figure 13).

\subsection{Generalized correlation proposed}

The results obtained for average Nusselt number in turbulent regime induced by natural convection within of a channel with symmetrical heating, including the effects of aspect ratio, sloping and convergence angles, can be collated by the following correlation:

$$
\mathrm{Nu}_{L}=C\left(\operatorname{Ra}_{L} \cos \gamma \cos \Theta\right)^{m}
$$

with $C=0.0354$ and $m=0.371$, for $10^{10} \leq \operatorname{Ra}_{L} \leq 10^{16}, 0^{\circ} \leq \gamma \leq 30^{\circ}, 0^{\circ} \leq \Theta \leq 60^{\circ}$, and for the wide range of values of $b / L$ studied.

\subsection{Considerations on influence of aspect ratio}

As aforementioned, the correlation (23) appears to be valid for the wide range of values of aspect ratios studied. However, somme comments over the influence of $b / L$ should be considered. Results obtained suggest, in the case of vertical parallel channel, for low values of 
aspect ratio, that the constant in origin $C$ of Equation (23) must increase slightly, and for high aspect ratio, the slope $m$ must also decrease slightly, always for a value of $\operatorname{Ra}_{L}$ high enough for each value of $b / L$ in order to consider the flow as totally turbulent. However, it has been preferred to propose a global correlation which fits satisfactorily all the ranges of $b / L, \gamma$ and $\Theta$ studied.

For an aspect ratio of 0.25 , the correlation proposed fits to the results obtained with a maximum error of $5 \%$ for any value of $\gamma$ and $\Theta$ considered. For a lower aspect ratio, $b / L=$ 0.03 , the proposed correlation reaches average deviations of $15 \%$ for $\gamma=\Theta=0^{\circ}$, decreasing below $5 \%$ from values of $\gamma$ and $\Theta$ of $30^{\circ}$.

As it has been mentioned, the proposed correlation is valid for a fully turbulent regime. Hence the error range specified correspond to $\mathrm{Ra}_{L} \geq 10^{10}$. For high aspect ratios, the turbulent flow is reached at lower Rayleigh numbers, for the same turbulence intensity imposed at the inlet of the channel. In this way, the deviation of numerical results with respect to the proposed correlation decreases. For low values of $I$ and also with low aspect ratios, the laminar regime is extended up to values of $\mathrm{Ra}_{L}$ relatively high, delaying the transition and leading a double trend in the logarithm function $\mathrm{Nu}_{L}=\mathrm{Nu}_{L}\left(\operatorname{Ra}_{L}\right)$, as explained previously. It has been proved that when increasing the turbulence intensity at the channel inlet, this brings forward the transition to turbulence for relatively low values of $\mathrm{Ra}_{L}$. This mentioned trends, have agreed both in Phoenics and Fluent codes, with average errors of 5\%. In Figure 8, it can observed that these differences are slightly higher in the transition region.

\section{Comparison with experimental prototypes}

The correlation obtained in this numerical investigation has been checked in two experimental prototypes which contain in their configuration convergent and sloping channels formed by heated plates. As it can be observed in Figures 14 and 15, the prototypes are installed outdoors in two building ceiling placed in Murcia (Spain). Both are based on the combination of reflective and absorbent flat plates to form inclined channels that act during the summer as solar chimneys. During the sunny days of summer the absorbent plates of the system capture the solar radiation. Whereas the upper plates are directly heated by the sun, the lower ones are heated by light reflection. Thus, both plates forming the channel are hotter than ambient temperature and a vertical air flow is produced by natural convection through it. Hence the 
system acts as a reduced and two-dimensional solar chimney. Below the channel a water flow is sprayed crosscurrent. A little part of the water is evaporated and the rest is cooled by adiabatic evaporation and recovered at lower temperature. These prototypes are described in detail in [33] and [34].

In the first prototype (Figure 14) the channels are characterized by a convergence angle of $\gamma=3.73^{\circ}$, a sloping angle of $\Theta=67^{\circ}$ and an aspect ratio of $b / L=0.09$. In the second prototype (Figure 15) the convergence angle is $\gamma=9.8^{\circ}$, sloping angle $\Theta=52^{\circ}$ and aspect ratio $b / L=0.117$. Taking into account that both prototypes are installed outdoors in a building ceiling, they are dominated, on one hand, by the vacuum caused by the wind in the top of the chimneys and, on the other hand, when the condition of the wind is calmed, they are governed by natural convection effects.

Figure 16 shows the comparisons of correlations proposed for plate systems, when $\gamma=$ $3.73^{\circ}, \Theta=67^{\circ}$ and $b / L=0.09$, with an experimental correlation proposed for the first prototype. The trends highlighted by the correlation for plate systems are similar to those experimentally calculated for this one. Within the first prototype functioning range, $\mathrm{Ra}^{*} \in$ $\left[2 \times 10^{4}-5 \times 10^{4}\right]$, the average discrepancies obtained for the average Nusselt number are around 1\%. In Figure 17, the same comparison for the second prototype is carried out, with $\gamma=9.8^{\circ}, \Theta=52^{\circ}$ and $b / L=0.117$. Once again, the tendencies are similar. In this case, within the functioning range of this second prototype, $\mathrm{Ra}^{*} \in\left[5 \times 10^{4}-2 \times 10^{5}\right]$, the average discrepancies obtained for the Nusselt number are around 5\%.

\section{Conclusions}

Flows induced by natural convection for high Rayleigh numbers in channels, for different convergence and centerline sloping angles, have been studied. Also, it has been solved cases for intermediate values of $\mathrm{Ra}$, simulating the laminar-turbulent transition. Most of cases solved was for isothermal symmetric heating conditions. The low-Reynolds $k-\omega$ turbulence model has been validated through experimental and numerical results proposed by Versteegh and Nieuwstadt [7], Rodrigues et al. [15], Miyamoto et al. [5], Habib et al. [25] and Said et al. [11]. It has been conferred a special attention to the mesh and the value of $y^{+}$near-wall, limited to a range of $0.1-0.5$. Numerical results obtained by means Fluent and Phoenics finite-volume codes are almost coincidental. Results obtained show a good agreement with 
those obtained by above authors. It has been obtained the average Nusselt number $\mathrm{Nu}_{L}$ for $\mathrm{Ra}_{L}$ in the range $10^{6} \leq \operatorname{Ra}_{L} \leq 10^{16}$, aspect ratios $0.03 \leq b / L \leq 0.25$, convergence angles $\gamma=0^{\circ}$, $3^{\circ}, 6^{\circ}, 9^{\circ}, 12^{\circ}, 15^{\circ}$ y $30^{\circ}$, and sloping angles $\Theta=0^{\circ}, 15^{\circ}, 30^{\circ}, 45^{\circ}$ and $60^{\circ}$.

The following concluding remarks can be made:

1. The results presented have been obtained with a low turbulence intensity $(I)$ at the inlet of the channel, equal to $2 \%$. Keeping this suitable level of initial turbulence for natural convective flows, it has been found a dependence with respect to aspect ratio $b / L$ not totally negligible. It has been proved that such a dependence decreases as the initial value of $I$ increases, as a consequence of the advance in the laminar-turbulent transition.

2. For isothermal symmetric heating conditions, channels with high aspect ratio, and for low values of $I$ at the channel inlet, it has been detected that the roughly limit $\operatorname{Ra}_{L}=10^{9}$ can be accepted as good for transition point from laminar to turbulent flow, as it can be found in the literature [29]. For low values of $b / L$, it has been detected that the transition to turbulence begins at $\mathrm{Ra}_{L}$ next to $10^{11}$.

3. In sloped channels, qualitatively it can be observed that as the sloping angle $\Theta$ increases, the turbulence intensity generated in the lower plate is higher than the turbulence intensity generated in the upper one. This fact leads to obtain an asymmetrically field of velocity in the channel exit. It has been proved that when tilting the channel a reduction of the buoyancy on the core of fluid occurs within the channel. This can be corrected including the factor $(\cos \Theta)$ in the definition of the Rayleigh number.

4. With regards to the influence of the convergence angle $\gamma$, it can be observed that the higher this parameter is the higher the turbulent kinetic energy is, giving place to an advancement in the transition from laminar to turbulent regime in the boundary layer. However, the effect of this advancement is small compared to the reduction of buoyancy produced by the inclination of the plates. It has been proved that there is an overlap of the results if $\mathrm{Ra}$ is modified through the factor $(\cos \gamma)$, in line with the previous work focused in laminar flow [16].

5. For sloped and convergent channels, the results for average Nusselt number can be fitted to those obtained for vertical channels by modifying the Rayleigh number through the factor $(\cos \Theta \cos \gamma)$. The following generalized correlation has been reported: $\mathrm{Nu}_{L}=$ 
$0.0354\left(\operatorname{Ra}_{L} \cos \gamma \cos \Theta\right)^{0.371}$, for $10^{10} \leq \operatorname{Ra}_{L} \leq 10^{16}, 0^{\circ} \leq \gamma \leq 30^{\circ}, 0^{\circ} \leq \Theta \leq 60^{\circ}$, and $0.03 \leq$ $b / L \leq 0.25$. There is a continuity between the correlation for laminar regime proposed in [16] and those obtained for turbulent regime in this study.

6. The numerical correlation obtained has been validated with experimental results for different prototypes. It is expected that this correlation can be applied to passive solar systems formed by plates (with vertical, sloped or converging configurations).

\section{Ackowledgement}

This research has been supported by the 'Dirección General de Investigación' of 'Ministerio de Educación y Ciencia' of Spanish Government, through DPI 2003-02719 Project. 


\section{Caption for table}

Table 1: Grid independence study for average Nusselt number as a function of $y^{+}$, for $\mathrm{Ra}^{*}=$ $10^{9}, b / L=0.1, \gamma=12^{\circ}$, and $\Theta=0^{\circ}$. Results obtained with Fluent, using the $k-\omega$ model. 


\section{Caption for figures}

Figure 1: Scheme of a converging sloped channel formed by isothermal AC and BD walls.

Figure 2: Influence of near-wall value of $y^{+}$on average Nusselt number, for a parallel vertical channel with $\operatorname{Ra}_{L}=10^{12}$ and $b / L=0.2$. Symmetric isothermal heating conditions. Results obtained with Phoenics. Grid of $200 \times 80$ cells. Case with $\gamma=\Theta=0$.

Figure 3: Comparison between non-dimensional average temperature profiles at wall, obtained by Versteegh and Nieuwstadt [7] with those obtained using Fluent and Phoenics in this work with the $k-\omega$ model. Isothermal walls asymmetrically heated at difference temperature of $39.1^{\circ} \mathrm{C} . \gamma=\Theta=0 . b / L=1 / 28.6$ and $b=0.076 \mathrm{~mm}$.

Figure 4: Distribution of wall temperature for a channel with a heat flux of $200 \mathrm{~W} / \mathrm{m}^{2}$ in a wall and $40 \mathrm{~W} / \mathrm{m}^{2}$ in the other wall $(L=2.5 \mathrm{~m}, b=0.3 \mathrm{~m})$, numerically obtained by Rodrigues et al. [15], and those obtained in this study. Case with $\gamma=\Theta=0$.

Figure 5: Comparison of velocity profiles obtained in this research (using Fluent and Phoenics codes) with those proposed by Miyamoto et al. [13] and those reached by Habib et al. [24] for a vertical channel with $\operatorname{Ra}_{L}=4 \times 10^{6}$, being $\bar{U}$ the average velocity in the channel. Cases with $\gamma=\Theta=0, L=0.125 \mathrm{~m}$ and $b=0.04 \mathrm{~m}$.

Figure 6: Distribution of the local Nusselt number based on $b$, along the wall isothermally heated in a vertical channel for different turbulence intensities, for $\mathrm{Ra}^{*}=1.6 \times 10^{6}, b / L=0.2$ and $\gamma=\Theta=0$. Results obtained with Phoenics.

Figure 7: Distribution of the local Nusselt number based on $b$, along the wall isothermally heated in a vertical channel for different turbulence intensities, for $\mathrm{Ra}^{*}=1.6 \times 10^{8}, b / L=0.2$ and $\gamma=\Theta=0$. Results obtained with Phoenics.

Figure 8: Average Nusselt number $\mathrm{Nu}_{L}$ as a function of the Rayleigh number $\mathrm{Ra}_{L}$, for different aspect ratios in a vertical channel, in turbulent regime. Cases with $\gamma=\Theta=0$. Laminar correlation proposed by Kaiser et al. [16] and isolated plate correlation proposed by Incropera and De Witt [29].

Figure 9: Average Nusselt number $\mathrm{Nu}_{L}$ as a function of the Rayleigh number $\mathrm{Ra}_{L}$ in a channel for different sloping angles. Results obtained with Fluent. Comparison with Said et al. [11] and Churchill and Chu [28] for $\gamma=0$. Laminar correlation of Kaiser et al. [16] is also presented. 
Figure 10: Influence of the converging angle $\gamma$ of the channel on the local heat transfer coefficient for a case with $\mathrm{Ra}^{*}=10^{8}, b / L=0.1$ and $\Theta=0^{\circ}$. Results obtained with Fluent.

Figure 11: Influence of the converging angle $\gamma$ of the channel on the average Nusselt number $\mathrm{Nu}_{L}$ as a function of $\mathrm{Ra}_{L}$, for $\Theta=0$ and $b / L=0.1$. Numerical correlation proposed in this study has been drawn.

Figure 12: Non-dimensional contours of turbulent kinetic energy $\left(k / \bar{U}^{2}\right)$, for $\mathrm{Ra}^{*}=10^{8}$, converging angle $\gamma=30^{\circ}$, sloping angles $\Theta=0^{\circ}$ and $60^{\circ}$, and $b / L=0.1$. Results obtained with Fluent.

Figure 13: Influence of the sloping angle on the average Nusselt number $\mathrm{Nu}_{L}$ as a function of $\mathrm{Ra}_{L}$, taking into account the factor $(\cos \gamma \cos \Theta)$, for $b / L=0.1$. Results obtained with Fluent.

Figure 14: First experimental prototype of the hydro-solar chimney, that includes an inclined convergent channel.

Figure 15: Second experimental prototype of the hydro-solar chimney, that includes an inclined convergent channel.

Figure 16: Comparison between the correlation proposed in this study for the average Nusselt number $\mathrm{Nu}_{b}$ for sloped and convergent channels, particularised for $\gamma=3.73^{\circ}, \Theta=67^{\circ}$ and $b / L=0.09$, with the experimental correlation $\left(\mathrm{Nu}_{b}=0.534\left(\mathrm{Ra}^{*} \cos \Theta\right)^{0.251}\right)$, proposed by Kaiser [34], for the hydro-solar chimney first prototype, considering no wind.

Figure 17: Comparison between the correlation proposed in this study for the average Nusselt number $\mathrm{Nu}_{b}$ for sloped and convergent channels, particularised for $\gamma=9.8^{\circ}, \Theta=52^{\circ}$ and $b / L=0.117$, with the experimental correlation, $\mathrm{Nu}_{b}=\left(\mathrm{Ra}^{*} \cos \Theta\right)^{0.2}$, proposed by Kaiser [34], for the hydro-solar chimney second prototype, considering no wind. 


\section{References}

[1] Lloyd J. R., Sparrow E. M. On the instability of natural convection flow on inclined plates. J. Fluid Mech. 1970; 42:465-70.

[2] Haaland S. E., Sparrow E. M. Wave instability of natural convection flow on inclined surfaces accounting for nonparallelism of the basic flow. ASME J. Heat Transfer 1973; 96:405-7.

[3] Chen T. S., Tzuoo K. L. Vortex instability of free convection flow over horizontal and inclined surfaces. ASME J. Heat Transfer 1982; 104:637-43.

[4] Tzuoo K. L., Chen T. S., Armaly B. F. Wave instability of natural convection flow on inclined surfaces. ASME J. Heat Transfer 1985; 107:107-11.

[5] Miyamoto M., Katoh Y., Kurima J. Turbulent free convection heat transfer from vertical parallel plates in air (heat transfer characteristics). NACSIS Electronic Library Service $1983 ; 1: 1-7$.

[6] Yuan X., Moser A., Suter P. Wall functions for numerical simulation of turbulent natural convection along vertical plates. Int. J. Heat Mass Transfer 1993; 36:4477-85.

[7] Versteegh T. A., Nieuwstadt F. T. A direct numerical simulation of natural convection between two infinite vertical differentially heated walls scaling laws and wall functions. Int. J. Heat Mass Transfer 1999; 42:3673-93.

[8] Henkes R. A. W. M., Hoogendorn C. J. Comparison exercise for computations of turbulent natural convection in enclosures. Numerical Heat Transfer 1995; 28:59-78.

[9] Peng S., Davison L. Computation of turbulent buoyant flows in enclosures with lowreynolds number $k-\omega$ models. Int. J. Heat Fluid Flow 1999; 20:172-84.

[10] Xu W., Chen Q., NieuwstadtF. T. M. A new turbulence model for near wall natural convection. Int. J. Heat Mass Transfer 1998; 41:3161-76.

[11] Said S. A. M., Habib M. A., Badr H. M., Anwar S. Turbulent natural convection between inclined isothermal plates. Computers and Fluids 2005; 34:1025-39. 
[12] Fedorov A. G., Viskanta R. Turbulent natural convection heat transfer in an asymmetrically heated, vertical parallel-plate channel. Int. J. Heat Mass Transfer 1997; 40:3849-60.

[13] Miyamoto M., Katoh Y., Kurima J., Suki H. Turbulent free convection heat transfer from vertical parallel plates. in Heat Transfer, Eds. C.L. Tien, V.P. Carey and J.K. Ferrel, Hemisphere, Washington D.C. 1986; 4:1593-98.

[14] La Pica A., Rodonò G., Volpes R. An experimental investigation on natural convection of air in a vertical channel. Int. J. Heat Mass Transfer 1993; 36:611-16.

[15] Rodrigues A. M., Canha A., Lahellec A., Grandpeix J. Y. Modelling natural convection in a heated vertical channel for room ventilation. Buildings and Environment 2000; 35:45569.

[16] Kaiser A. S., Zamora B., Viedma A. Correlation for nusselt number in natural convection in vertical convergent channels at uniform wall temperature by a numerical investigation. Int. J. Heat Fluid Flow 2004; 25:671-82.

[17] Philips J. R. Direct simulation of turbulent unstratified natural convection in a vertical slot for Pr $=0.71$. Int. J. Heat Mass Transfer 1996; 39:2485-94.

[18] Kasagi N., Nishimura M. Direct numerical simulation of combined forced and natural turbulent convection in a vertical plane channel. Int. J. Heat Fluid Flow 1997; 18:88-99.

[19] Kolmogorov A. N. Equations of turbulent motion of an incompresible fluid. Izvestia Academy of Sciences, USSR, Physics 1942; 6:56-58.

[20] Wilcox D. C. Turbulence Modeling for CFD. 2nd ed., DCW Industries; 2003.

[21] Fluent Inc. Fluent 6 manuals. Fluent Inc. ed.; 2006.

[22] Hernández J., Zamora B., Campo A. On the effect of Prandtl number and aspect ratio upon laminar natural-convection flows in vertical channels. Proc. Tenth International Heat Transfer Conference 1994; 5:483-88.

[23] Patankar S. V., Spalding D. B. A calculation procedure for heat, mass and momentum transfer in three-dimensional parabolic flows. Int. J. Heat Mass Transfer 1972; 15:1787806. 
[24] Van Leer B. Towards the ultimate conservative difference scheme V. A second order sequel to Gadunov's method. J. Comput. Phys. 1979; 32:101-36.

[25] Habib M. A., Said S. A. M., Ahmed S. A., Asghar A. Velocity characteristics of turbulent natural convection in symmetrically and asymmetrically heated vertical channels. Experimental Thermal of Fluid Science 2002; 26:77-87.

[26] Kitamura K. , Koike M., Fukuoka I. , Saito T. Large eddy structure and heat transfer of turbulent natural convection along a vertical flat plate. Int. J. Heat Mass Transfer 1995; 28:837-50.

[27] Vliet G. C., Liu C. K. An experimental study of natural convection boundary layers. Int. J. Heat Mass Transfer 1969; 91:517-31.

[28] Boudjemadi R., Maupu V., Laurence D., Le Quere P. Budgets of turbulent stresses and fluxes in a vertical slot natural convection flow at rayleigh $\mathrm{Ra}=10^{5}$ and $5.4 \times 10^{5}$. Int. J. Heat Fluid Flow 1997; 18:70-79.

[29] Churchill S. W., Chu H. H. S. Correlating equations for laminar and turbulent free convection from a vertical plate. Int. J. Heat Mass Transfer 1975; 18:1323.

[30] Incropera F. P., De Witt D. P. Introduction to Heat Transfer. Wiley and Sons; 1990.

[31] Azevedo L. F., Sparrow E. M. Natural convection in open-ended inclined channels. Int. J. Heat Mass Transfer 1985; 107:893-901.

[32] Biertümpfel R., Beer H. Natural convection heat transfer increase at the laminarturbulent transition in the presence of instationary longitudinal vortices. Int. J. Heat Mass Transfer 2003; 46:3109-17.

[33] Kaiser A. S., Viedma A. Hydrosolar roof for integrated energy dissipation and capture in buildings. Energy and Buildings 2001; 33:673-82.

[34] Lucas M., Martínez P., Sánchez A., Viedma A., Zamora B. Improved hydrsolar roof for buildings' air conditioning. Energy and Buildings 2003; 35:963-70.

[35] Kaiser A. S. Estudio de la transferencia de calor y de los flujos convectivos inducidos en una cubierta hídrico-solar. PhD thesis, Universidad Politécnica de Cartagena, Spain; 2005. 


\section{Tables}

\begin{tabular}{|c|c|c|c|}
\hline Cells & $y^{+}$ & $\mathrm{Nu}_{L}$ & Error (\%) \\
\hline 1,920 & 120.62 & 226.4 & 0.1 \\
\hline 4,416 & 74.35 & 254.0 & 12.0 \\
\hline 38,400 & 24.69 & 258.0 & 13.8 \\
\hline 59,712 & 13.29 & 261.3 & 15.3 \\
\hline 78,144 & 6.92 & 266.4 & 17.5 \\
\hline 127,296 & 1.58 & 215.1 & 5.1 \\
\hline 60,480 & 1.36 & 214.7 & 5.3 \\
\hline 60,480 & 0.81 & 219.5 & 3.2 \\
\hline 95,040 & 0.69 & 222.6 & 1.8 \\
\hline 141,120 & 0.35 & 225.8 & 0.4 \\
\hline 187,200 & 0.17 & 226.4 & 0.2 \\
\hline 279,360 & 0.09 & 226.7 & 0.0 \\
\hline
\end{tabular}

Table 1: Grid independence study for average Nusselt number as a function of $y^{+}$, for $\mathrm{Ra}^{*}=$ $10^{9}, b / L=0.1, \gamma=12^{\circ}$, and $\Theta=0^{\circ}$. Results obtained with Fluent, using the $k-\omega$ model. 


\section{Figures}

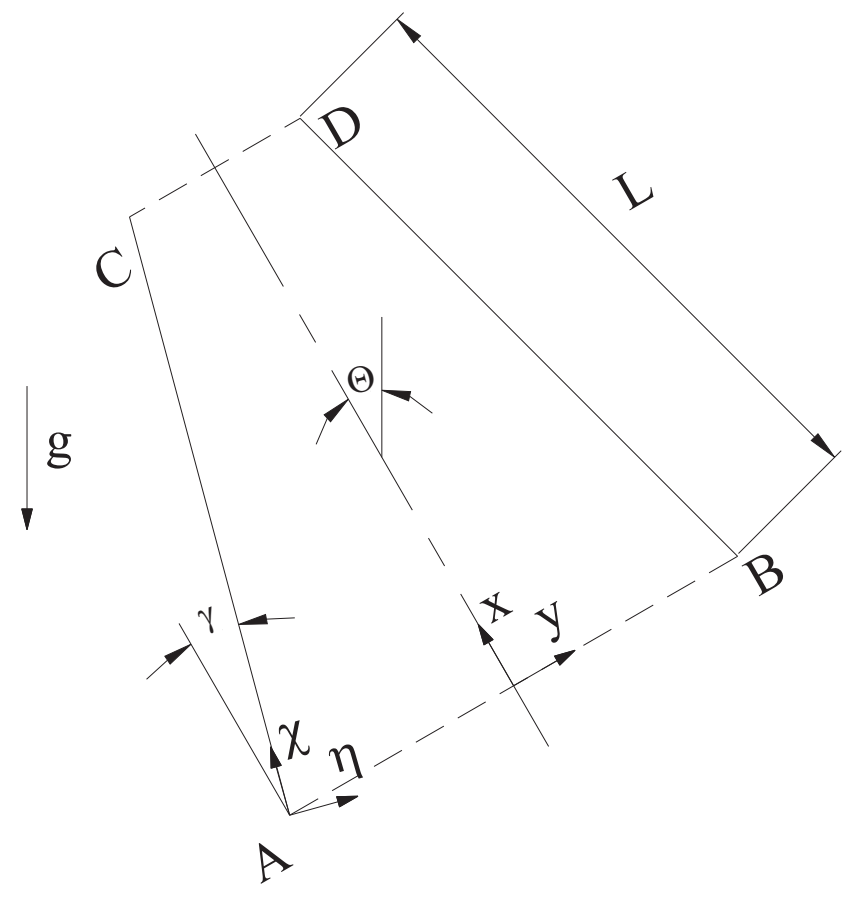

Figure 1: Scheme of a converging sloped channel formed by isothermal AC and BD walls. 


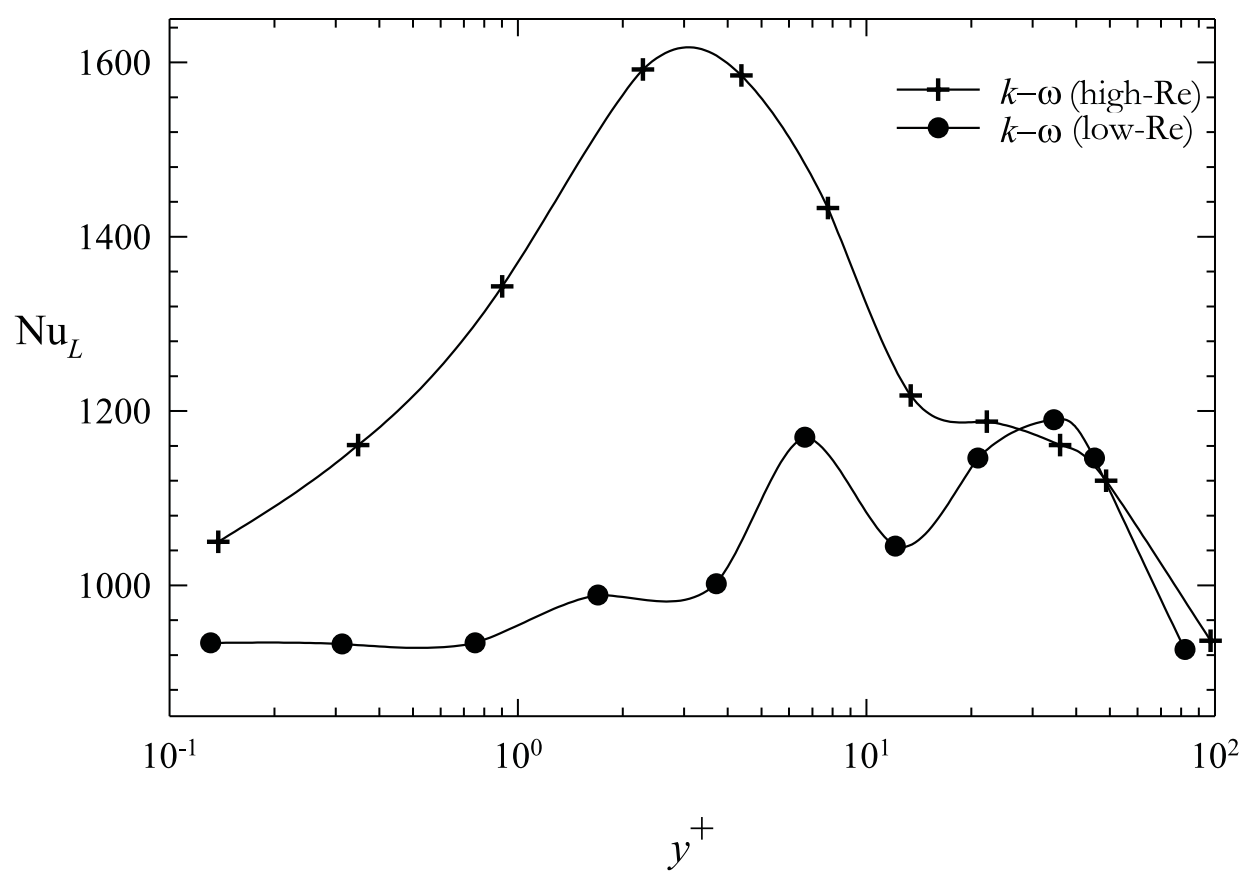

Figure 2: Influence of near-wall value of $y^{+}$on average Nusselt number, for a parallel vertical channel with $\mathrm{Ra}_{L}=10^{12}$ and $b / L=0.2$. Symmetric isothermal heating conditions. Results obtained with Phoenics. Grid of $200 \times 80$ cells. Case with $\gamma=\Theta=0$. 


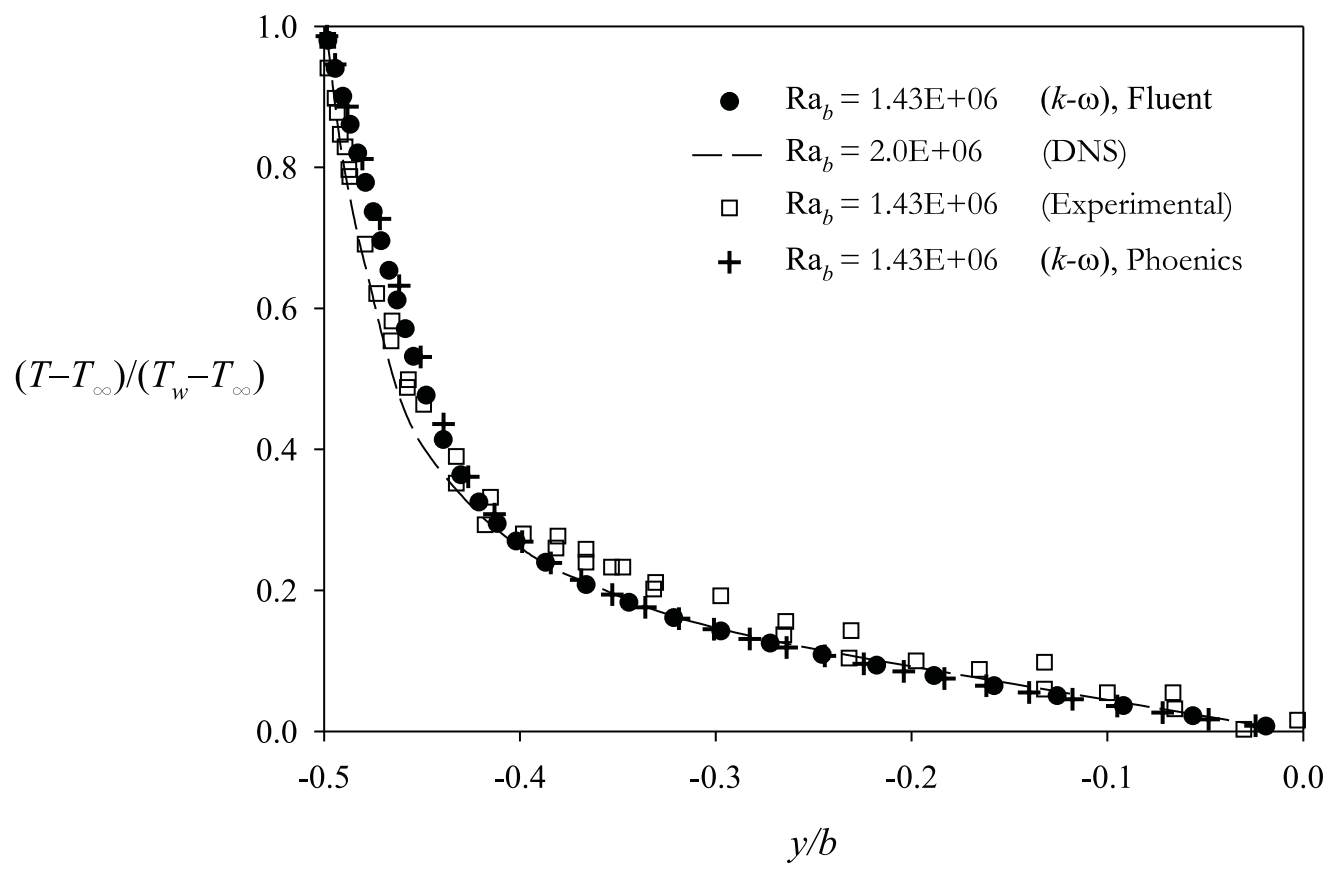

Figure 3: Comparison between non-dimensional average temperature profiles at wall, obtained by Versteegh and Nieuwstadt [7] with those obtained using Fluent and Phoenics in this work with the $k-\omega$ model. Isothermal walls asymmetrically heated at difference temperature of $39.1^{\circ} \mathrm{C} . \gamma=\Theta=0 . b / L=1 / 28.6$ and $b=0.076 \mathrm{~mm}$. 


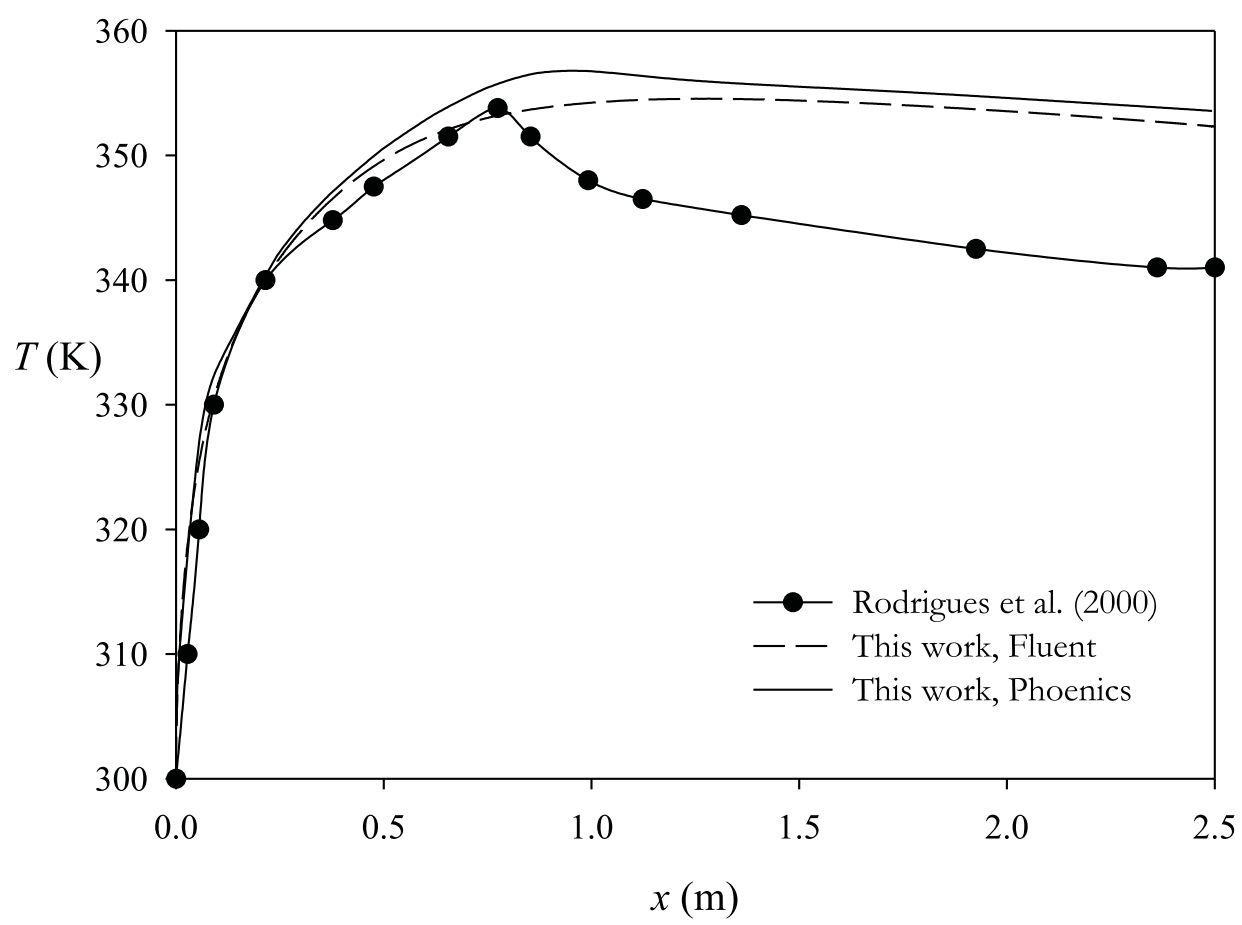

Figure 4: Distribution of wall temperature for a channel with a heat flux of $200 \mathrm{~W} / \mathrm{m}^{2}$ in a wall and $40 \mathrm{~W} / \mathrm{m}^{2}$ in the other wall $(L=2.5 \mathrm{~m}, b=0.3 \mathrm{~m})$, numerically obtained by Rodrigues et al. [15], and those obtained in this study. Case with $\gamma=\Theta=0$. 


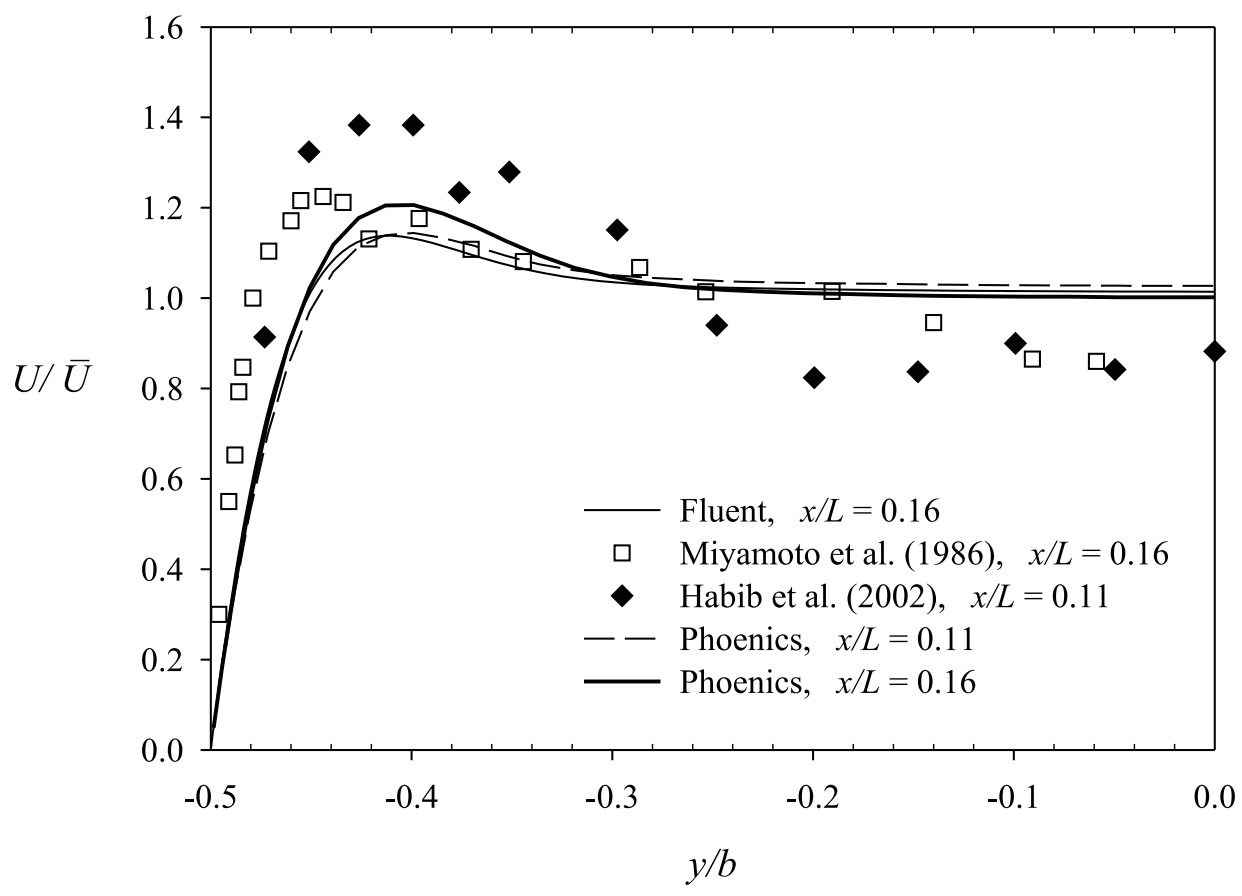

Figure 5: Comparison of velocity profiles obtained in this research (using Fluent and Phoenics codes) with those proposed by Miyamoto et al. [13] and those reached by Habib et al. [24] for a vertical channel with $\operatorname{Ra}_{L}=4 \times 10^{6}$, being $\bar{U}$ the average velocity in the channel. Cases with $\gamma=\Theta=0, L=0.125 \mathrm{~m}$ and $b=0.04 \mathrm{~m}$. 


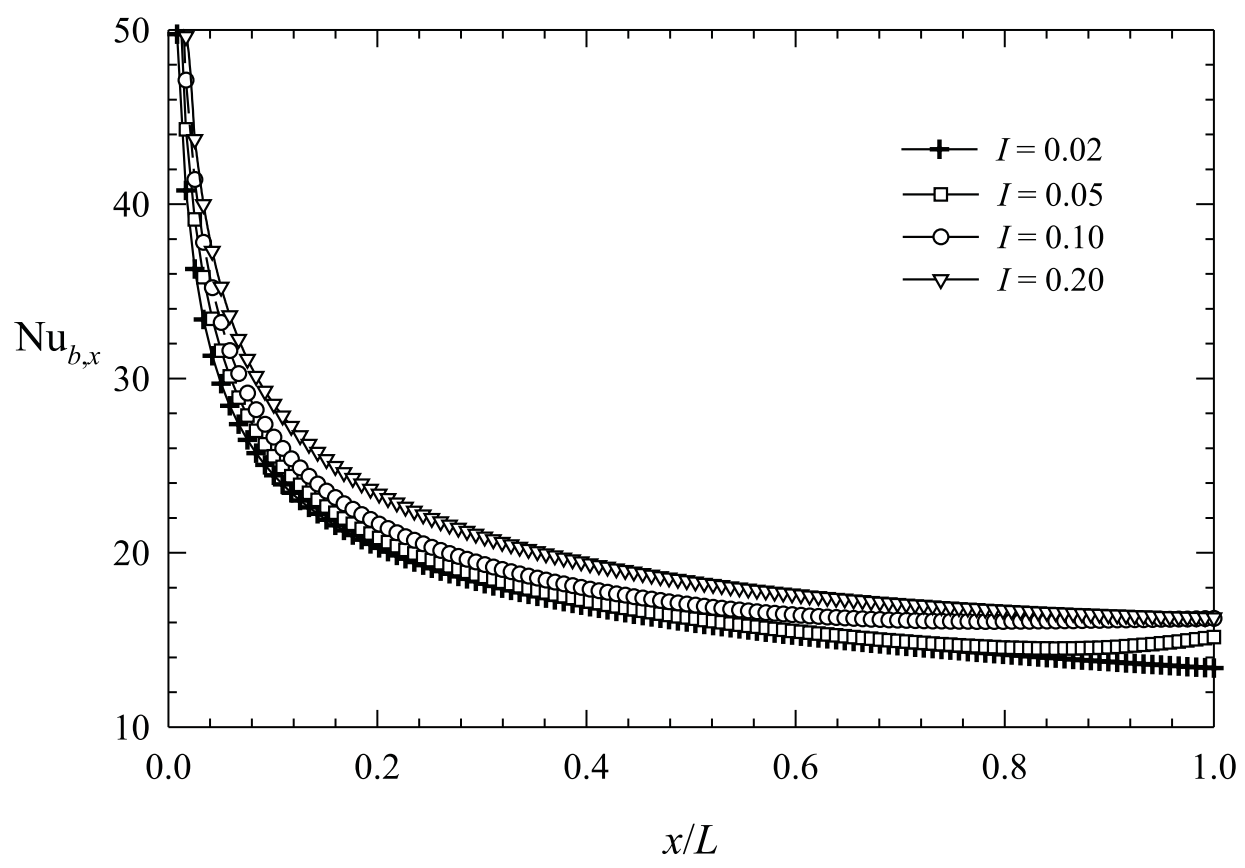

Figure 6: Distribution of the local Nusselt number based on $b$, along the wall isothermally heated in a vertical channel for different turbulence intensities, for $\mathrm{Ra}^{*}=1.6 \times 10^{6}, b / L=0.2$ and $\gamma=\Theta=0$. Results obtained with Phoenics. 


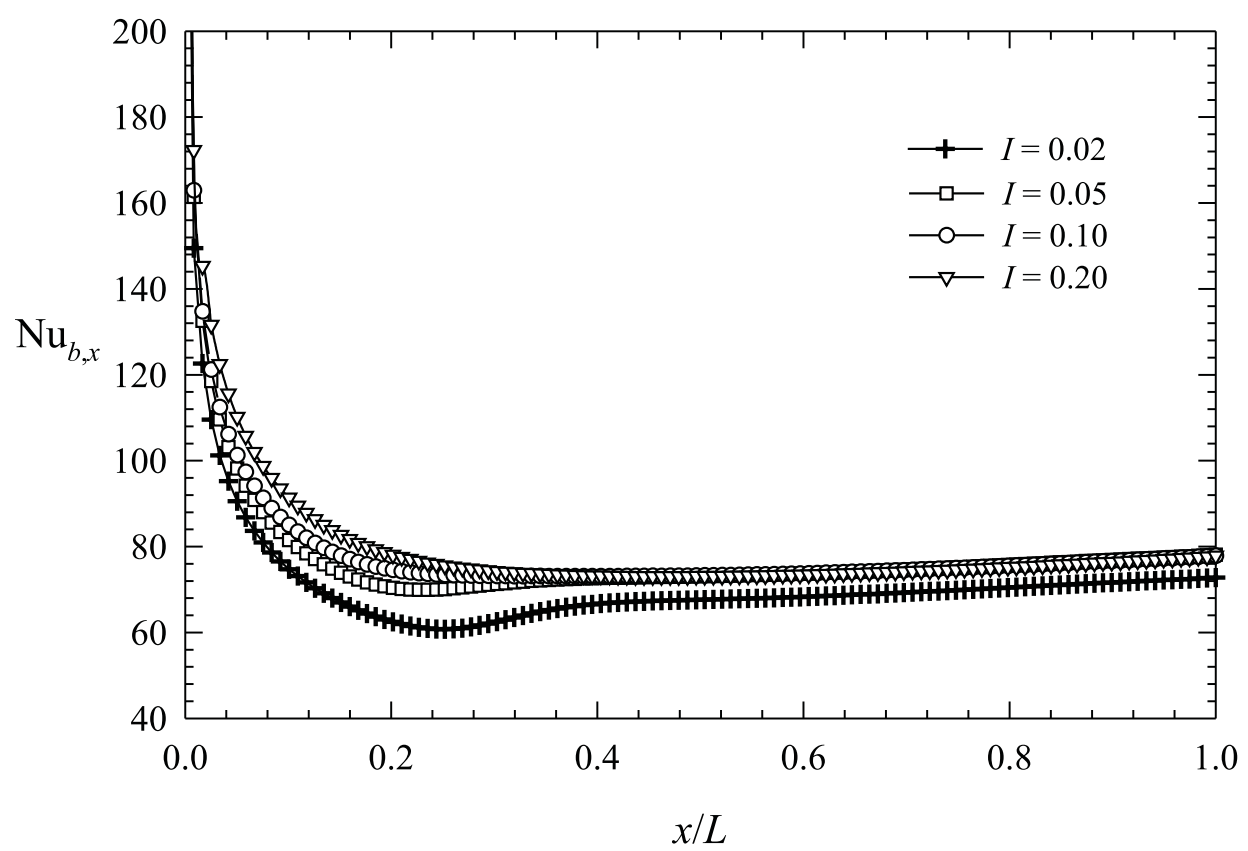

Figure 7: Distribution of the local Nusselt number based on $b$, along the wall isothermally heated in a vertical channel for different turbulence intensities, for $\mathrm{Ra}^{*}=1.6 \times 10^{8}, b / L=0.2$ and $\gamma=\Theta=0$. Results obtained with Phoenics. 


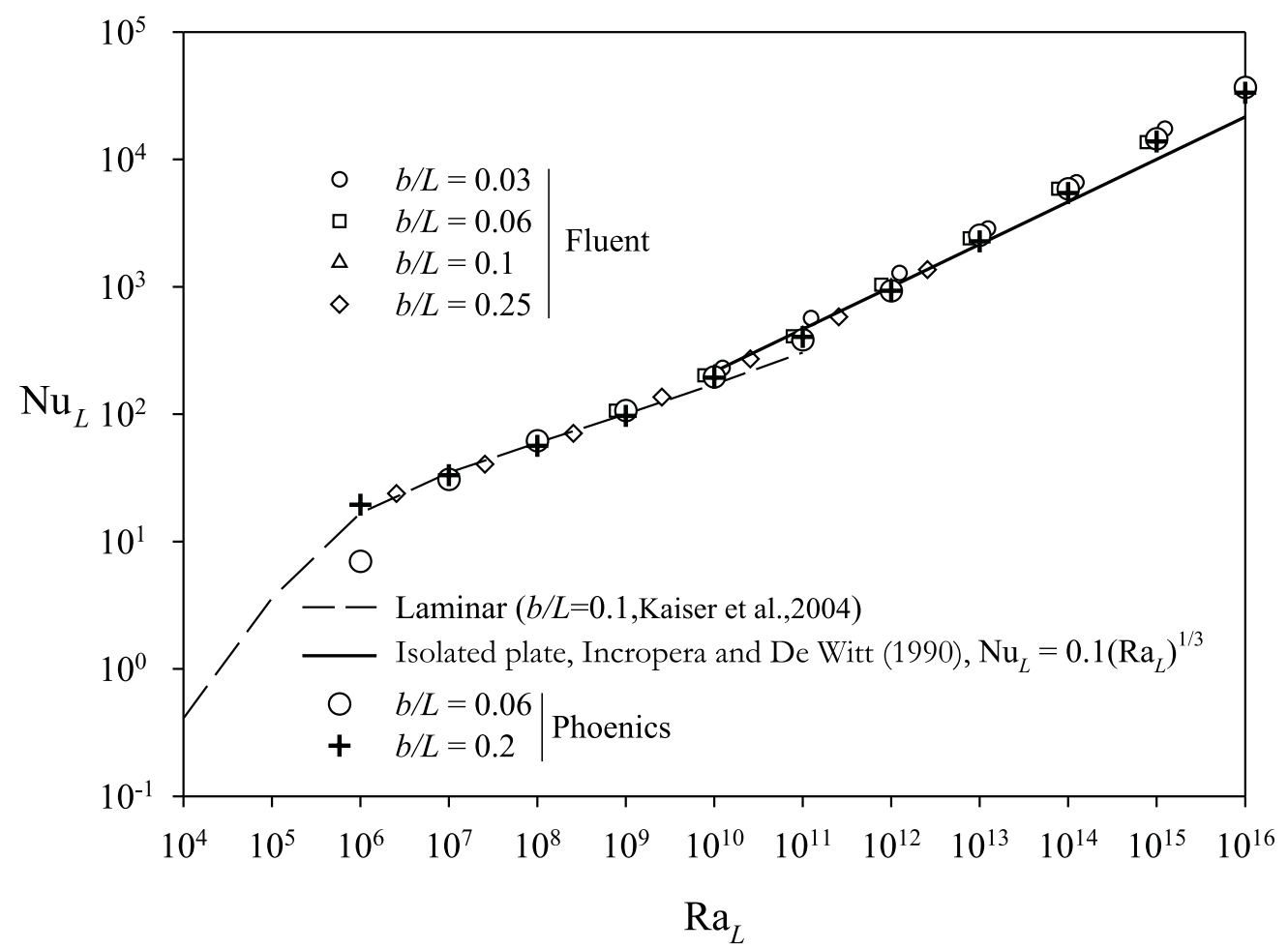

Figure 8: Average Nusselt number $\mathrm{Nu}_{L}$ as a function of the Rayleigh number $\mathrm{Ra}_{L}$, for different aspect ratios in a vertical channel, in turbulent regime. Cases with $\gamma=\Theta=0$. Laminar correlation proposed by Kaiser et al. [16] and isolated plate correlation proposed by Incropera and De Witt [29]. 


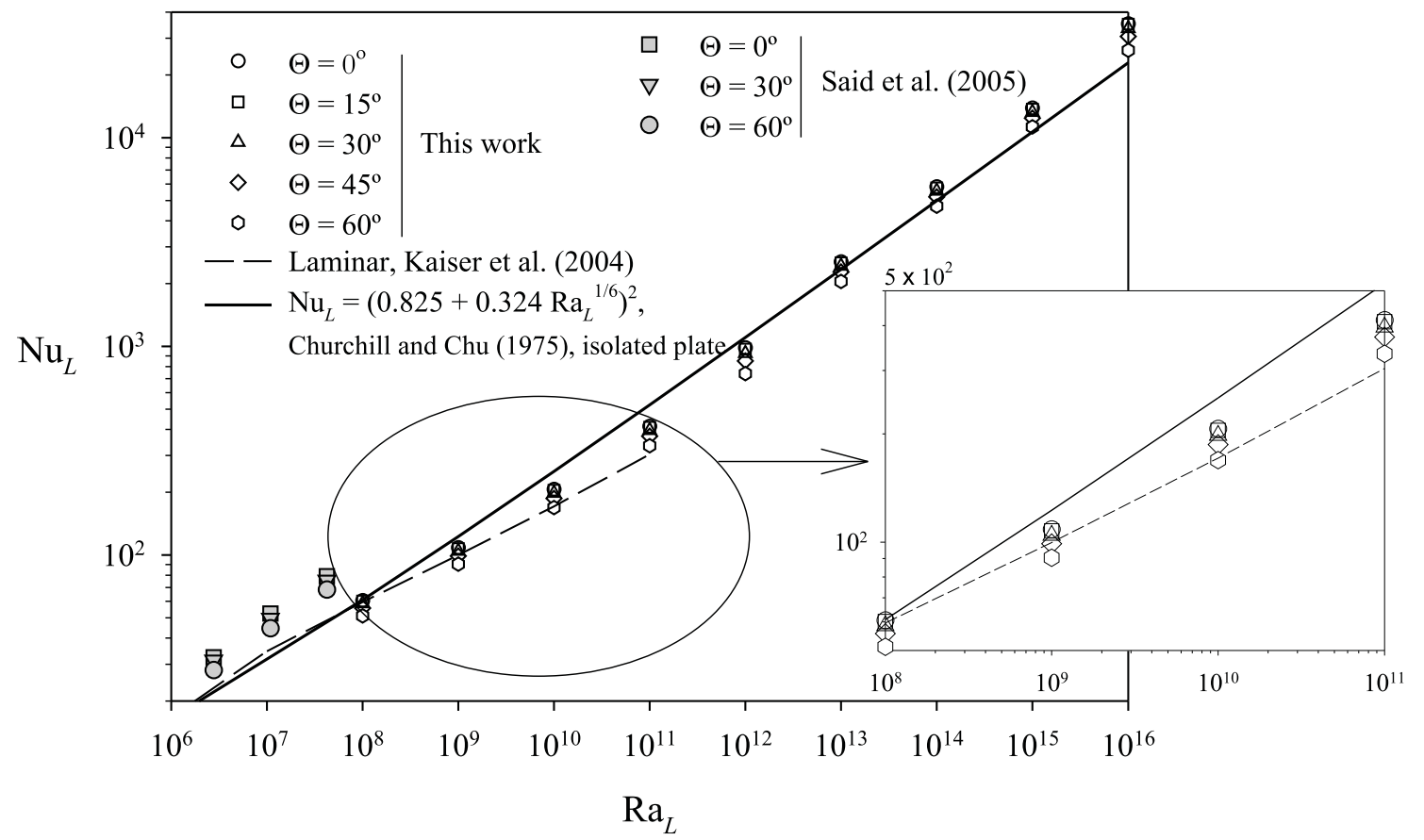

Figure 9: Average Nusselt number $\mathrm{Nu}_{L}$ as a function of the Rayleigh number $\mathrm{Ra}_{L}$ in a channel for different sloping angles. Results obtained with Fluent. Comparison with Said et al. [11] and Churchill and Chu [28] for $\gamma=0$. Laminar correlation of Kaiser et al. [16] is also presented. 


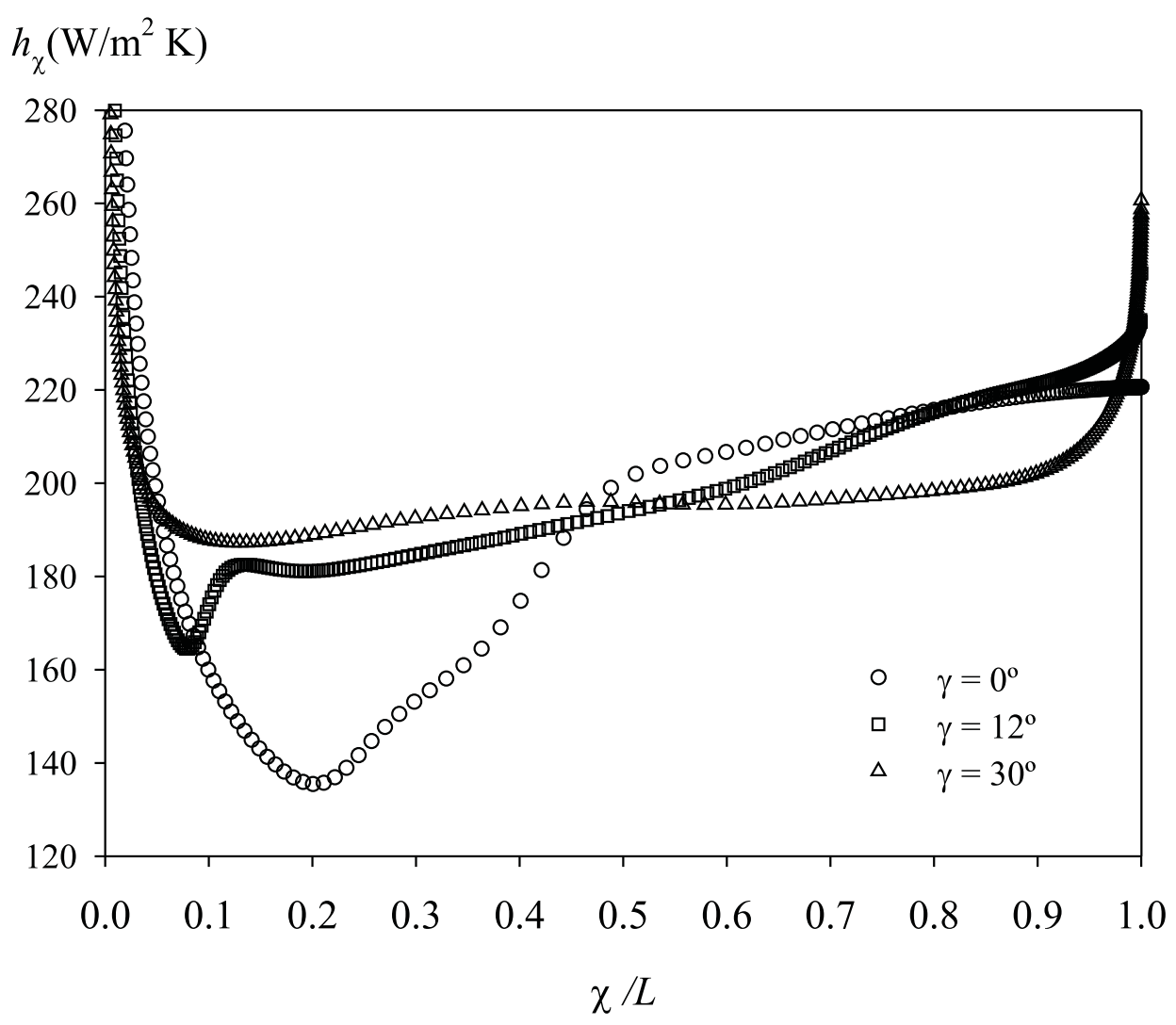

Figure 10: Influence of the converging angle $\gamma$ of the channel on the local heat transfer coefficient for a case with $\mathrm{Ra}^{*}=10^{8}, b / L=0.1$ and $\Theta=0^{\circ}$. Results obtained with Fluent. 


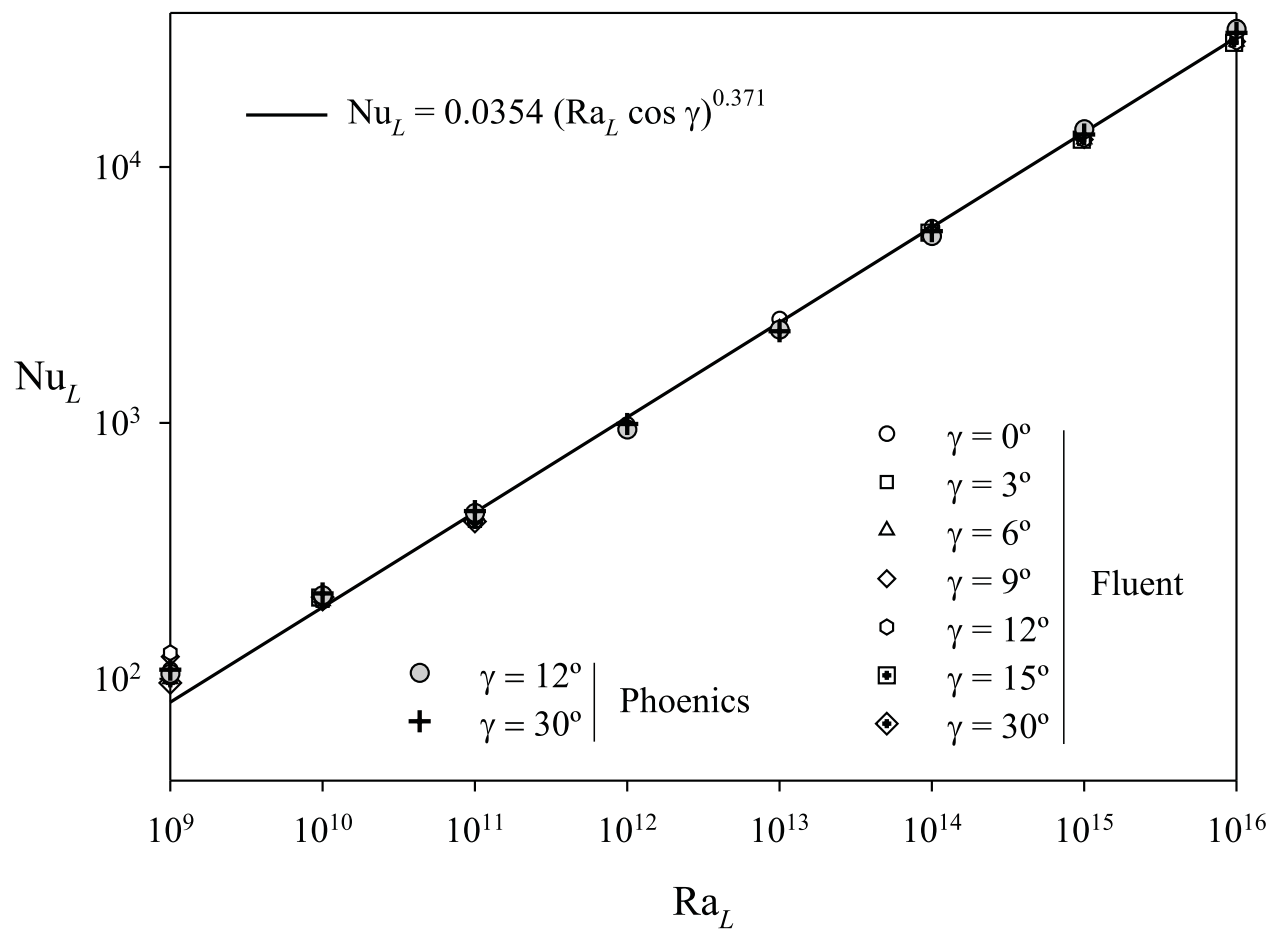

Figure 11: Influence of the converging angle $\gamma$ of the channel on the average Nusselt number $\mathrm{Nu}_{L}$ as a function of $\mathrm{Ra}_{L}$, for $\Theta=0$ and $b / L=0.1$. Numerical correlation proposed in this study has been drawn. 

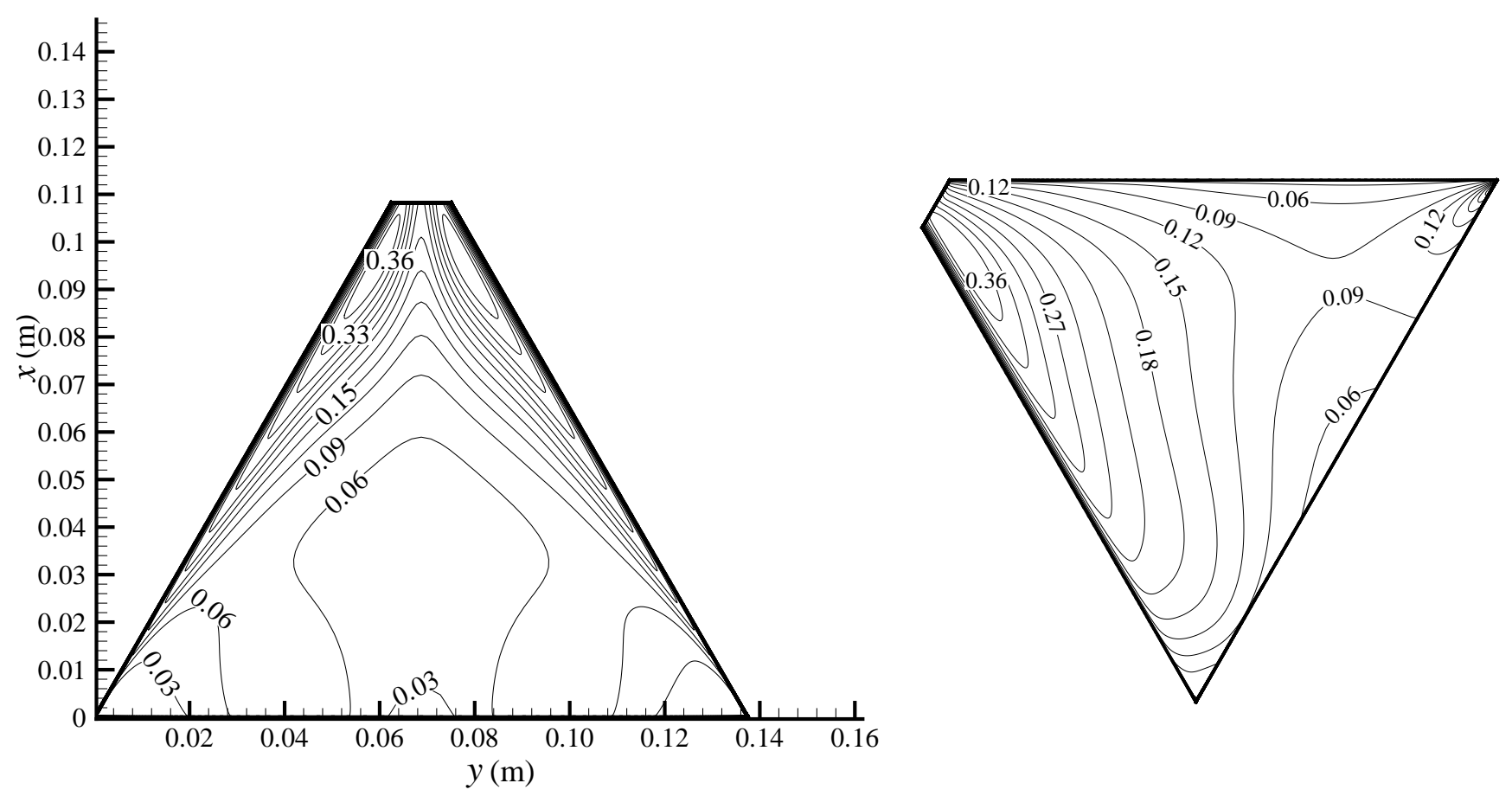

Figure 12: Non-dimensional contours of turbulent kinetic energy $\left(k / \bar{U}^{2}\right)$, for Ra* $=10^{8}$, converging angle $\gamma=30^{\circ}$, sloping angles $\Theta=0^{\circ}$ and $60^{\circ}$, and $b / L=0.1$. Results obtained with Fluent. 


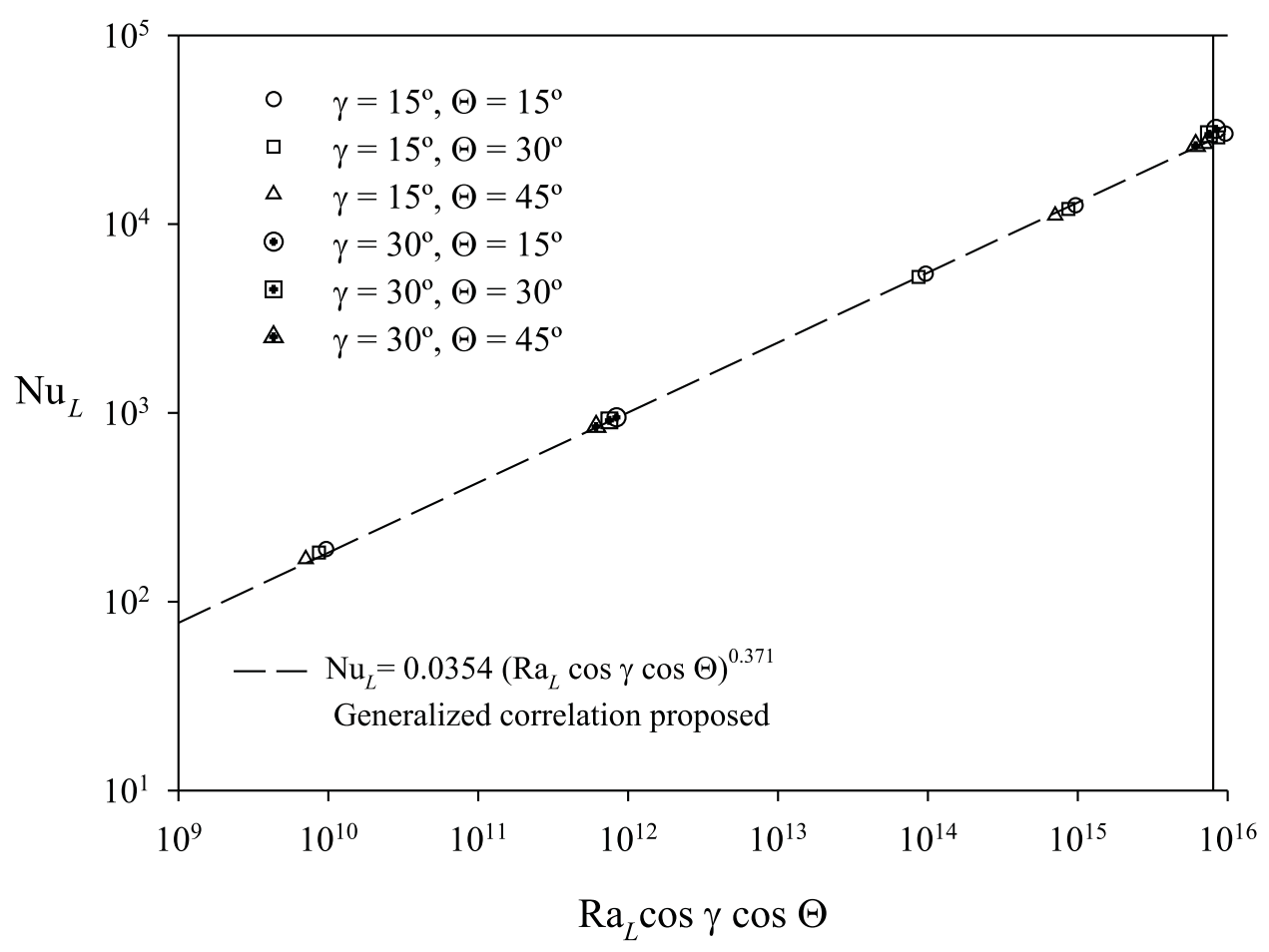

Figure 13: Influence of the sloping angle on the average Nusselt number $\mathrm{Nu}_{L}$ as a function of $\mathrm{Ra}_{L}$, taking into account the factor $(\cos \gamma \cos \Theta)$, for $b / L=0.1$. Results obtained with Fluent. 


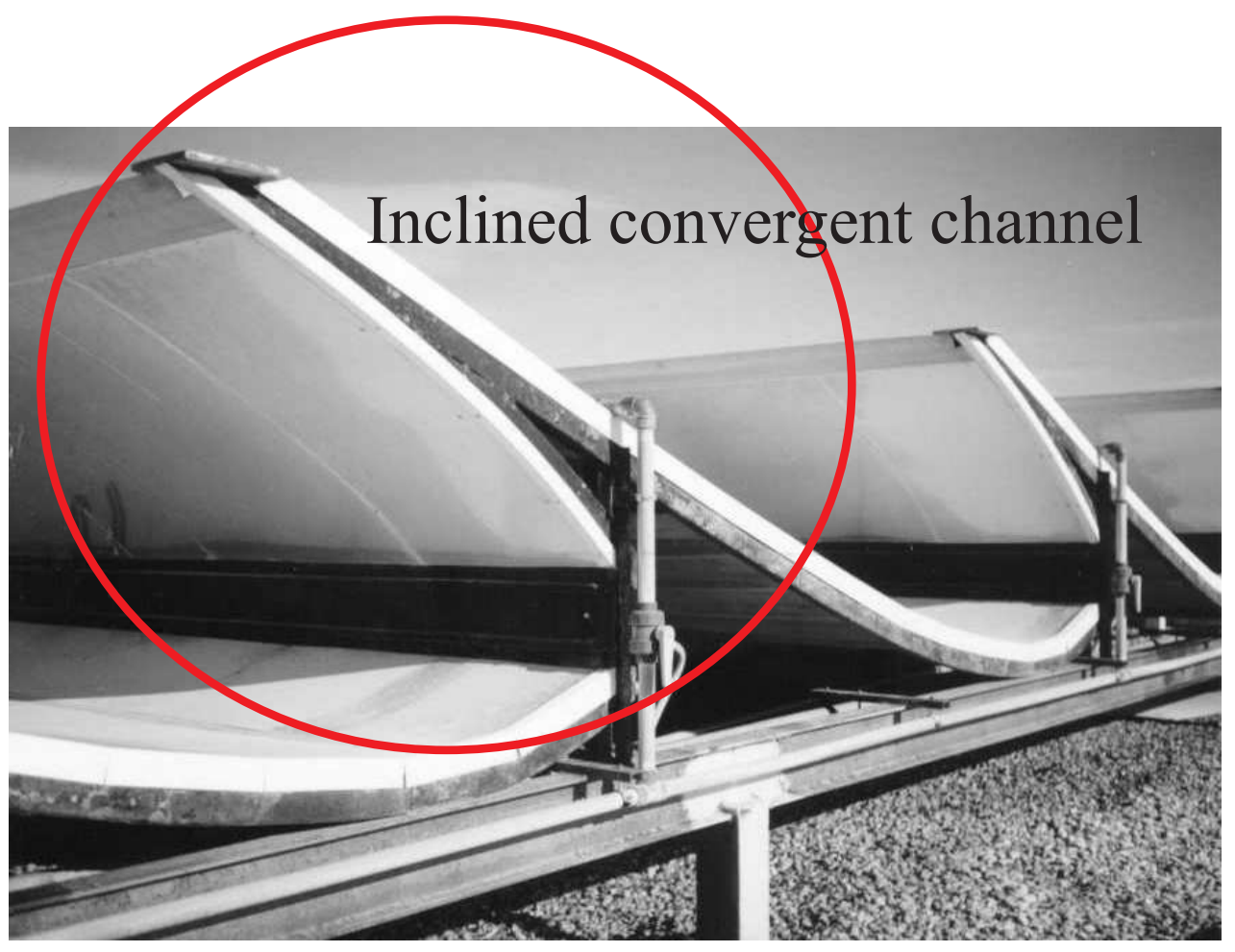

Figure 14: First experimental prototype of the hydro-solar chimney, that includes an inclined convergent channel. 


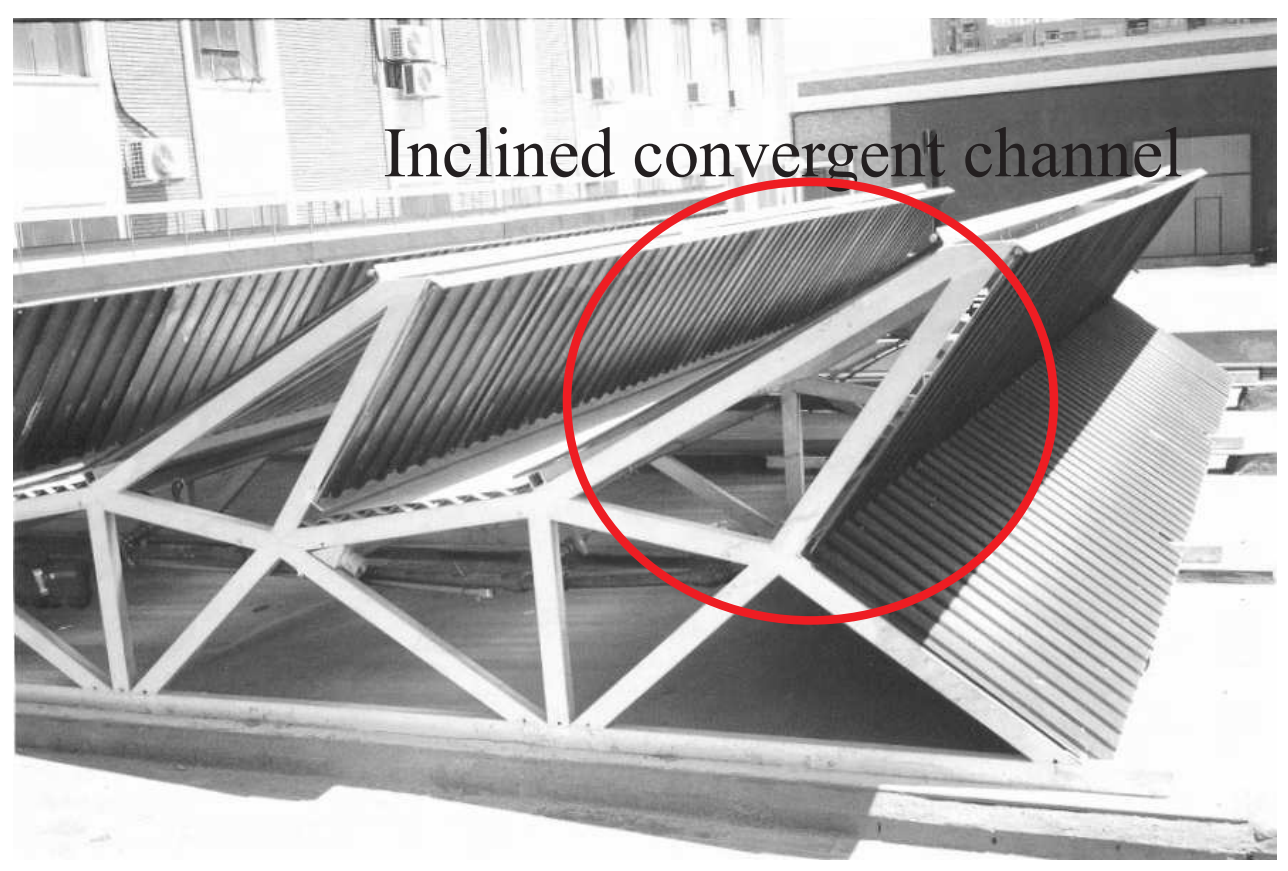

Figure 15: Second experimental prototype of the hydro-solar chimney, that includes an inclined convergent channel. 


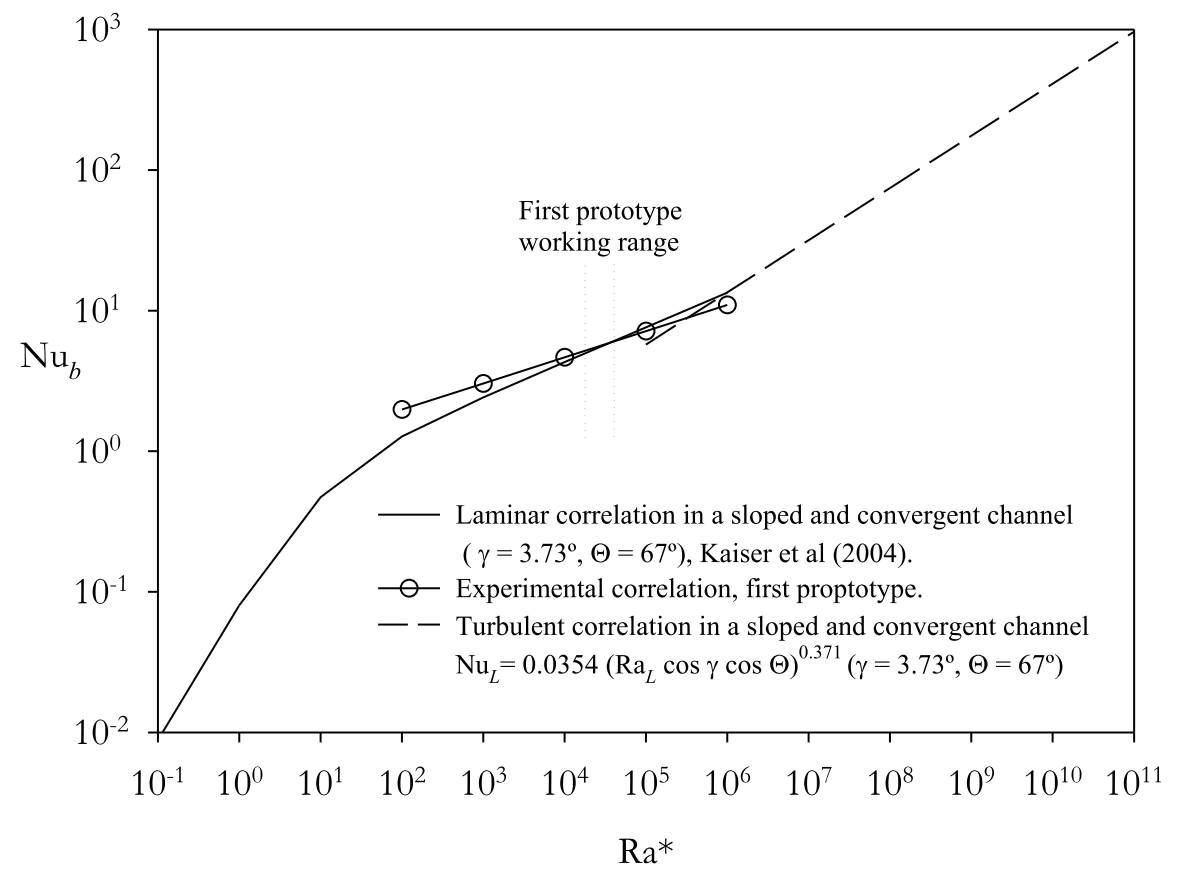

Figure 16: Comparison between the correlation proposed in this study for the average Nusselt number $\mathrm{Nu}_{b}$ for sloped and convergent channels, particularised for $\gamma=3.73^{\circ}, \Theta=67^{\circ}$ and $b / L=0.09$, with the experimental correlation $\left(\mathrm{Nu}_{b}=0.534\left(\mathrm{Ra}^{*} \cos \Theta\right)^{0.251}\right)$, proposed by Kaiser [34], for the hydro-solar chimney first prototype, considering no wind. 


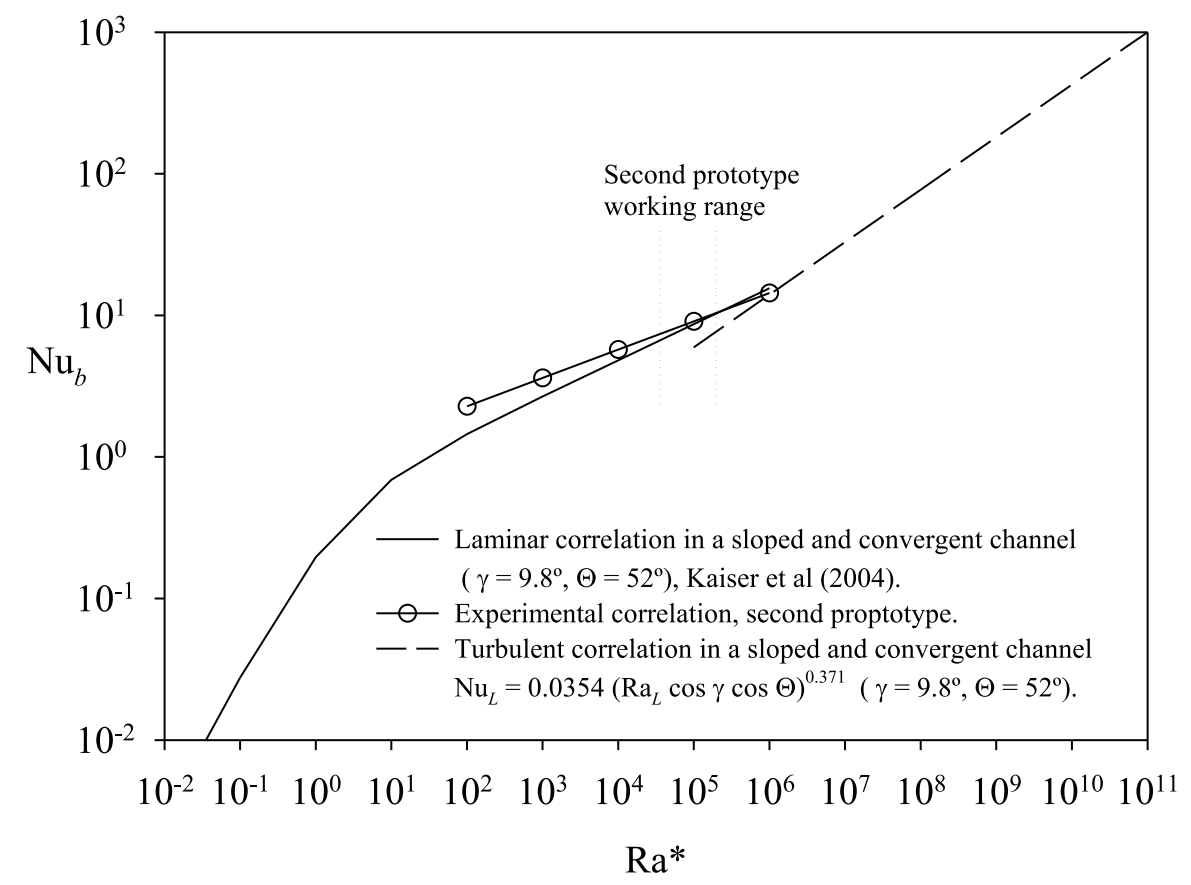

Figure 17: Comparison between the correlation proposed in this study for the average Nusselt number $\mathrm{Nu}_{b}$ for sloped and convergent channels, particularised for $\gamma=9.8^{\circ}, \Theta=52^{\circ}$ and $b / L=0.117$, with the experimental correlation, $\mathrm{Nu}_{b}=\left(\mathrm{Ra}^{*} \cos \Theta\right)^{0.2}$, proposed by Kaiser [34], for the hydro-solar chimney second prototype, considering no wind. 